\title{
Gravitational wave and collider searches for electroweak symmetry breaking patterns
}

\author{
Ruiyu Zhou, ${ }^{1}$ Ligong Bian $\odot,{ }^{1, *}$ Huai-Ke Guo, ${ }^{2, \dagger}$ and Yongcheng $\mathrm{Wu}^{3, \$}$ \\ ${ }^{1}$ Department of Physics, Chongqing University, Chongqing 401331, China \\ ${ }^{2}$ Department of Physics and Astronomy, University of Oklahoma, Norman, Oklahoma 73019, USA \\ ${ }^{3}$ Ottawa-Carleton Institute for Physics, Carleton University, \\ 1125 Colonel By Drive, Ottawa, Ontario K1S 5B6, Canada
}

(Received 29 November 2019; accepted 14 January 2020; published 10 February 2020)

\begin{abstract}
Utilizing the Georgi-Machacek model, we study the electroweak symmetry breaking mechanism with extra electroweak symmetry breaking contributions (eEWSB) that are bounded by the Fermi constant and limits from the related collider searches. The eEWSB is helpful to build a different zero temperature vacuum structure from the Standard Model (SM), and therefore leads to different electroweak phase transition patterns at the early Universe. We investigate the collider search prospects and gravitational waves (GW) predictions from the strongly firstly order phase transition (SFOEWPT) in this scenario. The Higgs pair searches at lepton colliders are found to be complementary with the GW searches of the SFOEWPT parameter spaces.
\end{abstract}

DOI: 10.1103/PhysRevD.101.035011

\section{INTRODUCTION}

The observation of the SM Higgs at $126 \mathrm{GeV}$ at LHC $[1,2]$ is a milestone of the particle physics, which means that the $W$ and $Z$ bosons obtain their masses through the electroweak symmetry breaking (EWSB) mechanism. The cubic and quartic Higgs couplings are supposed to be crucial to reveal the Higgs potential shape and the EWSB mechanism. The sensitivity of measurement of these couplings at LHC is pretty low, while future precision measurements are able to tell if there are new physics beyond the Standard Model (SM) that could drive deviation of the EWSB and how large the deviation could be. The observation of gravitational waves from the Binary Black Hole Merger by the LIGO and Virgo collaborations [3] opens a new era to search for fundamental physics. An important category of gravitational waves is a stochastic background [4] originated from the early Universe. One important source of this kind is a strongly first order electroweak phase transition (SFOEWPT), which gives a dynamical explanation of the EWSB as the Universe cools

\footnotetext{
*Corresponding author. lgbycl@cqu.edu.cn

ghk@ou.edu

Corresponding author. ycwu@physics.carleton.ca

Published by the American Physical Society under the terms of the Creative Commons Attribution 4.0 International license. Further distribution of this work must maintain attribution to the author(s) and the published article's title, journal citation, and DOI. Funded by SCOAP ${ }^{3}$.
}

down, and is a crucial ingredient in the explanation of the baryon asymmetry of the Universe within the framework of the electroweak baryogenesis mechanism (EWBG). ${ }^{1}$

New physics that takes part in the electroweak phase transition process may or may not contribute an extra component of EWSB contribution. For example, the SM plus real singlet model (xSM) has been extensively studied where the singlet scalar does not contribute to the EWSB (see Ref. [9] for a recent study). Meanwhile, the triplets in the Georgi-Machacek (GM) model, as will be studied in this work, can indeed contribute to the EWSB. Though both models share the same vacuum structure topology, the triplets contribution to the EWSB, i.e., the extra EWSB contribution, is bounded by the Fermi constant and gauge boson related collider searches. Therefore, one can expect different collider phenomenology, different SFOEWPT behaviors and thus different gravitational wave signal predictions for different amounts of the extra EWSB contributions.

The zero temperature vacuum structure with extra local minimum in addition to the electroweak vacuum could yield the possibility of multistep phase transition as well as one-step phase transition. In Refs. [10,11], the relation between the zero temperature potential difference and the

\footnotetext{
${ }^{1}$ The SFOEWPT is one of the three Shakharov conditions [5] that quenches the sphaleron process inside the bubbles and therefore preserves the baryon asymmetry generated (See Ref. [6] for a recent review on EWBG, and Ref. [7] for a recent review on cosmic phase transitions; Ref. [8] builds the connection between the sphaleron and gravitational wave.).
} 
SFOEWPT condition has been studied within the 2HDM. For previous studies of multistep phase transition and related vacuum structure at zero temperature, we refer to Refs. [9,12-20]. In this work, we use the Georgi-Machacek model to reveal that, the extra EWSB contribution can induce one-step or two-step SFOEWPT depending on the vacuum structure that has been studied previously by us in Ref [21]. The one-step SFOEWPT occurs with the symmetry change from $S U(2)_{L} \times S U(2)_{R}$ to the phase where electroweak symmetry is broken. The two-step SFOEWPT occurs with the first-step being the symmetry change of $S U(2)_{L} \times S U(2)_{R} \rightarrow S U(2)_{V}$, and the following second step being the dynamical breaking of the electroweak symmetry. In this work we improved the algorithm for the calculation of the critical order parameters of the phase transition. We further evaluate the gravitational wave signals being generated during the SFOEWPT. In comparison with the one-step situation, the gravitational wave signal spectrum generated from two-step SFOEWPT is found much easier to be probed by the projected spacebased interferometers, such as: LISA [22], BBO, DECIGO (Ultimate-DECIGO) [23], TianQin [24] and Taiji [25] programs. This is significantly different from the xSM case as was studied in Ref. [9], where the vacuum expectation value (VEV) of the extra singlet is more free from the limits of the collider searches.

This work is organized as follows: After a brief introduction of the GM model, the vacuum structure analysis and the phase transition calculational approach are given in Sec. II. In Sec. III, we show the relation between the phase transition and the collider phenomenology and demonstrate how these two interplay on the extra EWSB contributions. The gravitational wave signal predictions from the one-step and two-step SFOEWPT are investigated in Sec. IV. The collider search prospects for the SFOEWPT valid regions are addressed in Sec. V. We finally conclude in Sec. VI. Some details about the model are listed in the Appendix.

\section{THE EWPT DYNAMICS AND METHODOLOGY}

In this section, within the framework of the GM model, we first develop the methods for vacuum structure analysis and phase transition critical order parameter analysis, which can be applied to the phase transition analysis of one-step and two-step types that may be realized with many multiscalar models, such as: $\mathrm{xSM}, 2 \mathrm{HDM}, 2 \mathrm{HDM}+\mathrm{S}$, NMSSM, etc. In the GM model, at the critical temperature, the strongly first order phase transition condition could be fulfilled when the phase transition strength $v_{c} / T_{c} \equiv \sqrt{h_{\phi}\left(T_{C}\right)^{2}+8 h_{\xi}\left(T_{C}\right)^{2}} / T_{C} \geq 1[21,26]$.

\section{A. The GM model}

In the Georgi-Machacek model, there is one isospin doublet scalar field $\phi=\left(\phi^{+}, \phi^{0}\right)^{T}$ with hypercharge $\mathrm{Y}=\frac{1}{2}$, one complex isospin triplet scalar field $\chi=\left(\chi^{++}, \chi^{+}, \chi^{0}\right)^{T}$ with hypercharge $\mathrm{Y}=1$, and one real triplet $\xi=\left(\xi^{+}, \xi^{0},-\xi^{+*}\right)^{T}$ with hypercharge $\mathrm{Y}=0$. The custodial symmetry is introduced at tree level by imposing a global $\mathrm{SU}(2)_{L} \times \mathrm{SU}(2)_{R}$ symmetry upon the scalar potential. The most general scalar potential $V$ invariant under $S U(2)_{L} \times S U(2)_{R}$ is given by

$$
\begin{aligned}
& V(\Phi, \Delta) \\
& =\frac{1}{2} m_{1}^{2} \operatorname{tr}\left[\Phi^{\dagger} \Phi\right]+\frac{1}{2} m_{2}^{2} \operatorname{tr}\left[\Delta^{\dagger} \Delta\right]+\lambda_{1}\left(\operatorname{tr}\left[\Phi^{\dagger} \Phi\right]\right)^{2} \\
& \left.+\lambda_{2} \operatorname{tr}\left[\Delta^{\dagger} \Delta\right]\right)^{2}+\lambda_{3} \operatorname{tr}\left[\left(\Delta^{\dagger} \Delta\right)^{2}\right]+\lambda_{4} \operatorname{tr}\left[\Phi^{\dagger} \Phi\right] \operatorname{tr}\left[\Delta^{\dagger} \Delta\right] \\
& +\lambda_{5} \operatorname{tr}\left[\Phi^{\dagger} \frac{\sigma^{a}}{2} \Phi \frac{\sigma^{b}}{2}\right] \operatorname{tr}\left[\Delta^{\dagger} T^{a} \Delta T^{b}\right] \\
& +\mu_{1} \operatorname{tr}\left[\Phi^{\dagger} \frac{\sigma^{a}}{2} \Phi \frac{\sigma^{b}}{2}\right]\left(P^{\dagger} \Delta P\right)_{a b}+\mu_{2} \operatorname{tr}\left[\Delta^{\dagger} T^{a} \Delta T^{b}\right]\left(P^{\dagger} \Delta P\right)_{a b},
\end{aligned}
$$

where the fields are expressed in bimultiplet form

$\Phi \equiv\left(\begin{array}{cc}\phi^{0 *} & \phi^{+} \\ -\phi^{+*} & \phi^{0}\end{array}\right), \quad \Delta \equiv\left(\begin{array}{ccc}\chi^{0 *} & \xi^{+} & \chi^{++} \\ -\chi^{+*} & \xi^{0} & \chi^{+} \\ \chi^{++*} & -\xi^{+*} & \chi^{0}\end{array}\right)$,

$\sigma$ 's and $T$ 's are the $2 \times 2$ (Pauli matrices) and $3 \times 3$ matrix representations of the $S U(2)$ generators, respectively, while $P$ matrix, which transforms the fields back to Cartesian basis, is

$$
P=\frac{1}{\sqrt{2}}\left(\begin{array}{ccc}
-1 & i & 0 \\
0 & 0 & \sqrt{2} \\
1 & i & 0
\end{array}\right)
$$

The neutral components of each field can be parametrized by their real and imaginary parts:

$\phi^{0}=\frac{\nu_{\phi}+h_{\phi}+i a_{\phi}}{\sqrt{2}}, \quad \chi^{0}=\frac{\nu_{\chi}+h_{\chi}+i a_{\chi}}{\sqrt{2}}, \quad \xi^{0}=\nu_{\xi}+h_{\xi}$,

where $\nu_{\phi}, \nu_{\chi}$, and $\nu_{\xi}$ are the VEVs of $\phi^{0}, \chi^{0}$, and $\xi^{0}$, respectively. With only neutral components of this model, the potential reads:

$$
\begin{aligned}
V_{0}= & \frac{1}{2} m_{1}^{2} h_{\phi}^{2}+\frac{1}{2} m_{2}^{2}\left(h_{\xi}^{2}+h_{\chi}^{2}\right)+\lambda_{1} h_{\phi}^{4}+\lambda_{2}\left(h_{\xi}^{2}+h_{\chi}^{2}\right)^{2} \\
& +\frac{1}{2} \lambda_{3}\left(2 h_{\xi}^{4}+h_{\chi}^{4}\right)+\lambda_{4} h_{\phi}^{2}\left(h_{\xi}^{2}+h_{\chi}^{2}\right) \\
& +\frac{1}{4} \lambda_{5} h_{\phi}^{2} h_{\chi}\left(2 \sqrt{2} h_{\xi}+h_{\chi}\right) \\
& +\frac{1}{4} \mu_{1} h_{\phi}^{2}\left(h_{\xi}+\sqrt{2} h_{\chi}\right)+3 \mu_{2} h_{\xi} h_{\chi}^{2} .
\end{aligned}
$$


We can derive the EWSB vacuum through the minimization conditions:

$$
\frac{\partial V_{0}}{\partial h_{\phi}}=\frac{\partial V_{0}}{\partial h_{\chi}}=\frac{\partial V_{0}}{\partial h_{\xi}}=0,
$$

where the fields other than $\phi^{0}, \chi^{0}$, and $\xi^{0}$ take zero VEVs. In this paper, the solution satisfying the relation $v_{\chi}=\sqrt{2} v_{\xi}$ is selected, by which the EWSB vacuum maintains the diagonal $S U(2)_{V}$ symmetry. Thus the electroweak parameter $\rho_{\mathrm{EW}}=m_{W}^{2} /\left(m_{Z}^{2} \cos \theta_{w}^{2}\right)=1$ is established at the tree level. The $W$ and $Z$ boson masses from the EWSB give the constraint,

$$
\nu_{\phi}^{2}+8 \nu_{\xi}^{2} \equiv \nu^{2}=\frac{1}{\sqrt{2} G_{F}} \approx(246 \mathrm{GeV})^{2} .
$$

When $\nu_{\phi}, \nu_{\xi} \neq 0$, with the help of Eq. (6) (under the relation $\nu_{\chi}=\sqrt{2} \nu_{\xi}$ ), we could rewrite $m_{1}^{2}, m_{2}^{2}$ in terms of $\nu_{\phi}, \nu_{\xi}$ and other parameters in the Higgs potential as:

$$
\begin{gathered}
m_{1}^{2}=-4 \lambda_{1} \nu_{\phi}^{2}-6 \lambda_{4} \nu_{\xi}^{2}-3 \lambda_{5} \nu_{\xi}^{2}-\frac{3}{2} \mu_{1} \nu_{\xi} \\
m_{2}^{2}=-12 \lambda_{2} \nu_{\xi}^{2}-4 \lambda_{3} \nu_{\xi}^{2}-2 \lambda_{4} \nu_{\phi}^{2}-\lambda_{5} \nu_{\phi}^{2}-\mu_{1} \frac{\nu_{\phi}^{2}}{4 \nu_{\xi}}-6 \mu_{2} \nu_{\xi}
\end{gathered}
$$

There are 13 scalar fields in this model. After diagonalizing the mass matrices, the fields can be rewritten as the physical scalars (quintuple, triplet, and, singlet respectively)

$$
\begin{gathered}
H_{5}^{++}=\chi^{++}, \quad H_{5}^{+}=\frac{1}{\sqrt{2}}\left(\chi^{+}-\xi^{+}\right), \quad H_{5}^{0}=\sqrt{\frac{1}{3}} h_{\chi}-\sqrt{\frac{2}{3}} h_{\xi}, \\
H_{3}^{+}=-\cos \theta_{H} \phi^{+}+\sin \theta_{H} \frac{1}{\sqrt{2}}\left(\chi^{+}+\xi^{+}\right), \\
H_{3}^{0}=-\cos \theta_{H} a_{\phi}+\sin \theta_{H} a_{\chi}, \\
h=\cos \alpha h_{\phi}-\frac{\sin \alpha}{\sqrt{3}}\left(\sqrt{2} h_{\chi}+h_{\xi}\right), \\
H_{1}=\sin \alpha h_{\phi}+\frac{\cos \alpha}{\sqrt{3}}\left(\sqrt{2} h_{\chi}+h_{\xi}\right),
\end{gathered}
$$

and the Goldstone bosons

$$
\begin{aligned}
G^{+} & =\sin \theta_{H} \phi^{+}+\cos \theta_{H} \frac{1}{\sqrt{2}}\left(\chi^{+}+\xi^{+}\right), \\
G^{0} & =\sin \theta_{H} a_{\phi}+\cos \theta_{H} a_{\xi},
\end{aligned}
$$

where $\sin \theta_{H}=\frac{2 \sqrt{2} \nu_{\xi}}{\nu}$ and $\cos \theta_{H}=\frac{\nu_{\phi}}{\nu}$, with $\theta_{H}$ parametrizing the isospin triplets contribution to the EWSB. The $\alpha$ is the mixing angle of the two singlets and is determined by the mass matrix of these scalars as will be shown below.

The 3 Goldstone bosons eventually become the longitudinal components of the $W$ and $Z$ bosons, while the remaining 10 physical fields can be organized into a quintuple $H_{5}=\left(H_{5}^{++}, H_{5}^{+}, H_{5}^{0}, H_{5}^{-}, H_{5}^{--}\right)^{T}$, a triplet $H_{3}=$ $\left(H_{3}^{+}, H_{3}^{0}, H_{3}^{-}\right)^{T}$, and two singlets $h$ and $H_{1}$, where the former $(h)$ is used to denote the SM-like Higgs boson. The triplet scalar is $C P$-odd, while others are $C P$-even. The masses of different multiplets can be written as

$$
\begin{aligned}
m_{H_{5}}^{2}= & m_{H_{5}^{ \pm \pm}}^{2}=m_{H_{5}^{ \pm}}^{2}=m_{H_{5}^{0}}^{2}=\left(8 \lambda_{3} \nu_{\xi}^{2}-\frac{3}{2} \lambda_{5} \nu_{\phi}^{2}\right) \\
- & \frac{\mu_{1} \nu_{\phi}^{2}}{4 \nu_{\xi}}-12 \mu_{2} \nu_{\xi}, \\
& m_{H_{3}}^{2}=m_{H_{3}^{ \pm}}^{2}=m_{H_{3}^{0}}^{2}=-\left(\frac{\lambda_{5}}{2}+\frac{\mu_{1}}{4 \nu_{\xi}}\right) \nu^{2} .
\end{aligned}
$$

The masses of the singlets $\left(m_{h, H_{1}}\right)$ are the eigenvalues of the mass matrix:

$$
M^{2}=\left(\begin{array}{ll}
M_{11}^{2} & M_{12}^{2} \\
M_{12}^{2} & M_{22}^{2}
\end{array}\right),
$$

with

$$
\begin{gathered}
M_{11}^{2}=8 \cos ^{2} \theta_{H} \lambda_{1} \nu^{2}, \\
M_{22}^{2}=\sin ^{2} \theta_{H}\left(3 \lambda_{2}+\lambda_{3}\right) \nu^{2}+\cos ^{2} \theta_{H} M_{1}^{2}-\frac{1}{2} M_{2}^{2}, \\
M_{12}^{2}=\sqrt{\frac{3}{2}} \sin \theta_{H} \cos \theta_{H}\left[\left(2 \lambda_{4}+\lambda_{5}\right) \nu^{2}-M_{1}^{2}\right],
\end{gathered}
$$

where $M_{1}^{2}=-\frac{\nu}{\sqrt{2} \sin \theta_{H}} \mu_{1}$ and $M_{2}^{2}=-3 \sqrt{2} \sin \theta_{H} \nu \mu_{2}$. The mixing angle $\alpha_{H}$ is determined by

$$
\tan 2 \alpha_{H}=\frac{2 M_{12}^{2}}{M_{22}^{2}-M_{11}^{2}},
$$

as a function of $\theta_{H}$.

\section{B. On the vacuum structures and the possible EWPT patterns}

The leading order zero temperature effective potential of the GM model is given by Eq. (5). For the vacuum structure studies, we impose $h_{\chi}=\sqrt{2} h_{\xi}$ as required by the custodial symmetry, which ensures $\rho_{\mathrm{EW}}=1$ at the leading order. The general vacuum structure determined by the above potential is shown in Fig. 1, where A is the $\left(h_{\phi}, h_{\xi}\right)=(0,0)$ vacuum, $\mathrm{B}$ is the desired EW vacuum, $C_{1,2}$ are the alternative vacuums with $h_{\phi}=0$ [the $S U(2)_{V}$ vacuum]. Here, we use 


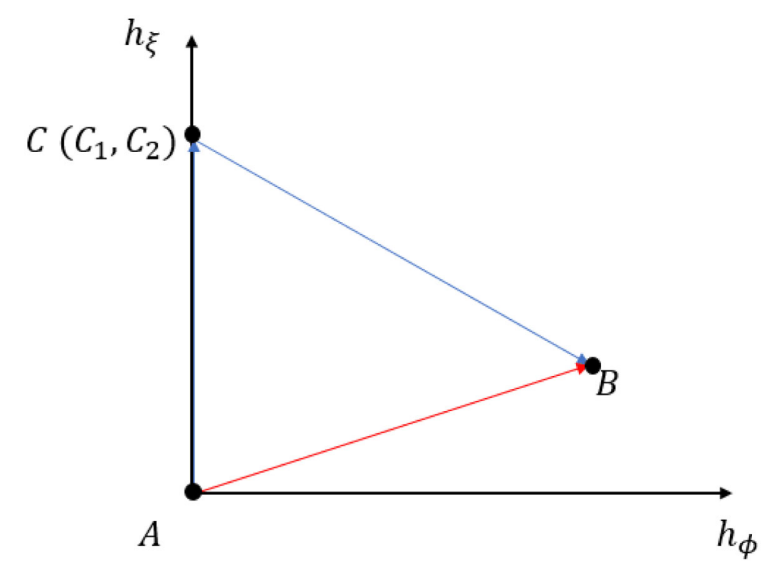

FIG. 1. The vacuum structure in the GM model.

$C$ to indicate $C_{1}$ and/or $C_{2}$ depending on which point is allowed by the positiveness of the Hessian matrix as will be explored below. In this paper we consider the case where there are two possible $\mathrm{C}$ points in the GM model which can be expressed as below,

$C_{1(2)}$ point: $h_{\phi} \rightarrow 0$,

$$
h_{\xi} \rightarrow \frac{-3 \mu_{2} \pm \sqrt{-12 m_{2}^{2} \lambda_{2}-4 m_{2}^{2} \lambda_{3}+9 \mu_{2}^{2}}}{4\left(3 \lambda_{2}+\lambda_{3}\right)} .
$$

The scalar potential at the EW vacuum (B) should be the global minimum one, and the value of the scalar potential at the original point is the maximal one of these three. The scalar potential at these three different vacuum points, $V_{0}(A), V_{0}(B), V_{0}\left(C_{1(2)}\right)$, are

$$
\begin{aligned}
V_{0}(A)= & 0, \\
V_{0}(B)= & -\lambda_{1} \nu_{\phi}^{4}-3 \nu_{\xi}^{3}\left(\mu_{2}+\left(3 \lambda_{2}+\lambda_{3}\right) \nu_{\xi}\right) \\
& -\frac{3}{8} \nu_{\xi}\left(\mu_{1}+4(2 \lambda 4+\lambda 5) \nu_{\xi}\right) \nu_{\phi}^{2}, \\
V_{0}\left(C_{1(2)}\right)= & -\frac{3}{256 \nu_{\xi}\left(3 \lambda_{2}+\lambda_{3}\right)^{3}} \\
& \times\left(F \mp 3 \mu_{2}\right)^{2}\left(\mu_{2}\left(24 \nu_{\xi}^{2}\left(3 \lambda_{2}+\lambda_{3}\right) \mp 2 \nu_{\xi} F\right)\right. \\
& +\left(3 \lambda_{2}+\lambda_{3}\right)\left(16 \nu_{\xi}^{3}\left(3 \lambda_{2}+\lambda_{3}\right)\right. \\
& \left.\left.+\nu_{\phi}^{2}\left(4 \nu_{\xi}\left(2 \lambda_{4}+\lambda_{5}\right)+\mu_{1}\right)\right)+6 \mu_{2}^{2} \nu_{\xi}\right), \\
\text { where } F= & \left(\frac{\nu_{\phi}^{2}\left(3 \lambda_{2}+\lambda_{3}\right)\left(4 \nu_{\xi}\left(2 \lambda_{4}+\lambda_{5}\right)+\mu_{1}\right)}{\nu_{\xi}}\right. \\
& \left.+\left(4 \nu_{\xi}\left(3 \lambda_{2}+\lambda_{3}\right)+3 \mu_{2}\right)^{2}\right)^{1 / 2} .
\end{aligned}
$$

The one-step phase transition would take place when $V_{0}(A)>V_{0}(B)\left(\Delta V_{0}(A B)>0\right)$ and $-12 m_{2}^{2} \lambda_{2}-4 m_{2}^{2} \lambda_{3}+$ $9 \mu_{2}^{2}<0$. Meanwhile, the two-step phase transition might happen when $-12 m_{2}^{2} \lambda_{2}-4 m_{2}^{2} \lambda_{3}+9 \mu_{2}^{2} \geq 0$ and
$V_{0}(A)>V_{0}\left(C_{1(2)}\right)>V_{0}(B) \quad\left(\right.$ with $\quad \Delta V_{0}\left(A C_{1(2)}\right)>0$, $\left.\Delta V_{0}\left(C_{1(2)} B\right)>0\right)$. The potential differences are given by the following:

$$
\begin{aligned}
\Delta V_{0}(A B) \equiv & V_{0}(A)-V_{0}(B) \\
= & \lambda_{1} \nu_{\phi}^{4}+3 \nu_{\xi}^{3}\left(\mu_{2}+\left(3 \lambda_{2}+\lambda_{3}\right) \nu_{\xi}\right) \\
& +\frac{3}{8} \nu_{\xi}\left(\mu_{1}+4\left(2 \lambda_{4}+\lambda_{5}\right) \nu_{\xi}\right) \nu_{\phi}^{2},
\end{aligned}
$$

$$
\begin{aligned}
\Delta & V_{0}\left(A C_{1(2)}\right) \\
\equiv & V_{0}(A)-V_{0}\left(C_{1(2)}\right) \\
= & \frac{3}{256 \nu_{\xi}\left(3 \lambda_{2}+\lambda_{3}\right)^{3}}\left(F \mp 3 \mu_{2}\right)^{2}\left(\mu _ { 2 } \left(24 \nu_{\xi}^{2}\left(3 \lambda_{2}+\lambda_{3}\right)\right.\right. \\
& \left.\mp 2 \nu_{\xi} F\right)+\left(3 \lambda_{2}+\lambda_{3}\right)\left(16 \nu \xi^{3}\left(3 \lambda_{2}+\lambda_{3}\right)\right. \\
& \left.\left.+\nu_{\phi}^{2}\left(4 \nu_{\xi}\left(2 \lambda_{4}+\lambda_{5}\right)+\mu_{1}\right)\right)+6 \mu_{2}^{2} \nu_{\xi}\right), \\
\Delta & V_{0}\left(C_{1(2)} B\right) \\
\equiv & V_{0}\left(C_{1(2)}\right)-V_{0}(B) \\
= & -\frac{3}{256 \nu_{\xi}\left(3 \lambda_{2}+\lambda_{3}\right)^{3}}\left(F \mp 3 \mu_{2}\right)^{2}\left(\mu _ { 2 } \left(24 \nu_{\xi}^{2}\left(3 \lambda_{2}+\lambda_{3}\right)\right.\right. \\
& \left.\mp 2 \nu_{\xi} F\right)+\left(3 \lambda_{2}+\lambda_{3}\right)\left(16 \nu_{\xi}^{3}\left(3 \lambda_{2}+\lambda_{3}\right)\right. \\
& \left.+\nu_{\phi}^{2}\left(4 \nu_{\xi}\left(2 \lambda_{4}+\lambda_{5}\right)+\mu_{1}\right)\right) \\
& \left.+6 \mu_{2}^{2} \nu_{\xi}\right)+\lambda_{1} \nu_{\phi}^{4}+3 \nu_{\xi}^{3}\left(\mu_{2}+\left(3 \lambda_{2}+\lambda_{3}\right) \nu_{\xi}\right) \\
& +\frac{3}{8} \nu_{\xi}\left(\mu_{1}+4\left(2 \lambda_{4}+\lambda_{5}\right) \nu_{\xi}\right) \nu_{\phi}^{2}, \\
& \quad \Delta V_{0}\left(C_{1} C_{2}\right) \equiv V_{0}\left(C_{1}\right)-V_{0}\left(C_{2}\right) \\
& =\frac{3 \mu_{2} F^{3}}{16\left(3 \lambda_{2}+\lambda_{3}\right)^{3} .}
\end{aligned}
$$

The $\Delta V_{0}\left(C_{1} C_{2}\right)$ determines the detailed phase transition patterns in the two-step phase transition scenario, as will be explored latter. Here, we note that one of the two configurations at $\mathrm{C}$ point is missed in Ref. [21]. The additional one configuration considered here opens a little more twostep SFOEWPT parameter space that passes the current constraints from the same-sign $W W$ channel search at $13 \mathrm{TeV}$ LHC [27], which highly restricts the triplet contribution to the EWSB.

We note that, as in the 2HDM case, to ensure the photon is massless, we do not expect the violation of the U(1) electromagnetic symmetry during the EWPT process and assume the classical values for the charged scaler fields to be zero [15,28-30]. Furthermore, we avoid all other possible alternative minima (see Ref. [31] for detail) by scanning model parameters with GMCalc [32], since the desired $\mathrm{EW}$ vacuum at the zero temperature (the $\mathrm{B}$ point in Fig. 1) is the electroweak-breaking and custodial SU(2)preserving minimum. 

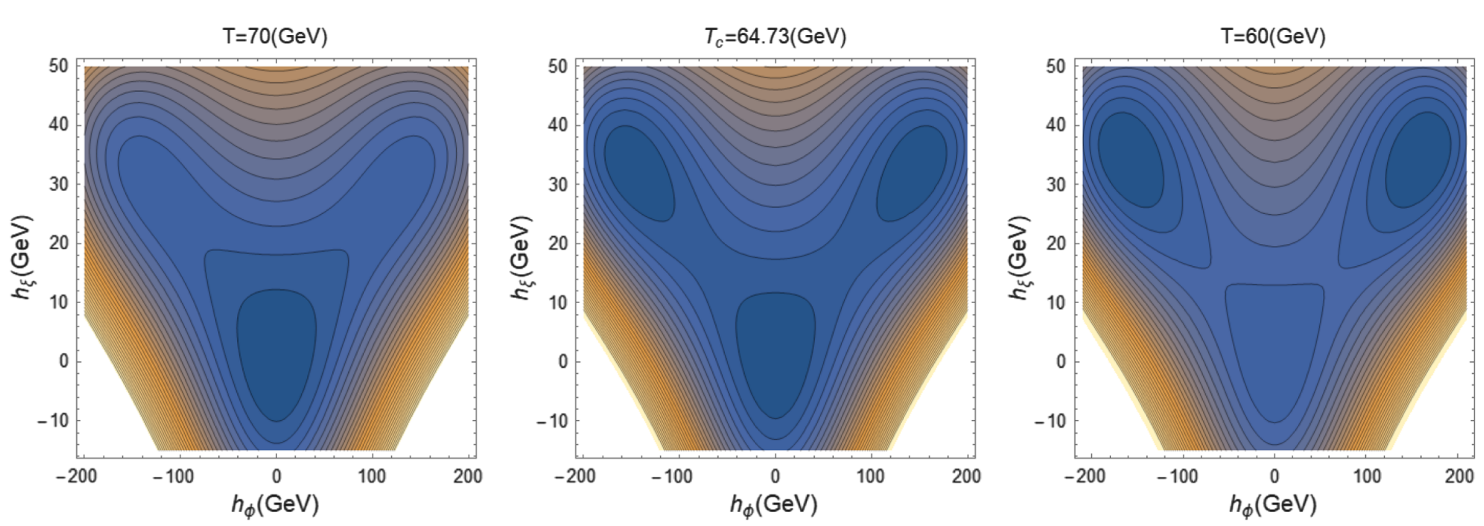

FIG. 2. The contours of $V_{T}$ in the $h_{\phi}-h_{\xi}$ plane, with the parameters being: $\lambda_{1}=0.035, \lambda_{2}=0.574, \lambda_{3}=-0.547, \lambda_{4}=0.798$, $\lambda_{5}=1.908, \theta_{H}=0.273, \mu_{1}=-360.373 \mathrm{GeV}, \mu_{2}=-47.377 \mathrm{GeV}$.

\section{Phase transition dynamics}

With the zero temperature scalar potential at hand, the phase transition dynamics can be estimated with the finite temperature potential using the gauge invariant approach that is equivalent to keeping the thermal mass corrections when using the high temperature expansion [33],

$$
V_{T}=V_{0}+\frac{1}{2} c_{\phi} T^{2} h_{\phi}^{2}+\frac{1}{2} c_{\xi} T^{2} h_{\xi}^{2}+\frac{1}{2} c_{\chi} T^{2} h_{\chi}^{2}
$$

with $V_{0}$ given in Eq. (5), and the finite temperature corrections being

$$
\begin{aligned}
& c_{\phi}=\frac{3 g^{2}}{16}+\frac{g^{\prime 2}}{16}+2 \lambda_{1}+\frac{3 \lambda_{4}}{2}+\frac{1}{4} y_{t}^{2} \sec ^{2} \theta_{H}, \\
& c_{\xi}=\frac{g^{2}}{2}+\frac{11 \lambda_{2}}{3}+\frac{7 \lambda_{3}}{3}+\frac{2 \lambda_{4}}{3}, \\
& c_{\chi}=\frac{g^{2}}{2}+\frac{g^{\prime 2}}{4}+\frac{11 \lambda_{2}}{3}+\frac{7 \lambda_{3}}{3}+\frac{2 \lambda_{4}}{3} .
\end{aligned}
$$

Here, we note that the traditional approach for studying the phase transition dynamics is to include four ingredients: the tree level potential, the Coleman-Weinberg potential [35], the finite temperature corrections, and the daisy resummation or the ring contribution [36]. For renormalizable theory, the potential barrier that drives the electroweak phase transition can come from the thermal loop corrections or tree level cubic terms [37]. The cubic term of $\mu_{1,2}$ [see Eq. (5)] from the tree level potential provides the barrier that drives the phase transition dynamics at the critical temperature and thus belongs to the second class. In this scenario, the gauge invariant approach we adopt here keeps the crucial cubic terms and can provide a transparent physical intuition as in Ref. [38], though additional SFOEWPT parameter space may open up when one includes the full four ingredients.

For the safety of custodial symmetry, we assume $h_{\chi}=$ $\sqrt{2} h_{\xi}$ for phase transition studies. The deviation from the

\footnotetext{
${ }^{2}$ Here, we note that the gauge dependence is small for gauges that are consistent with the perturbative expansion when one performs the phase transition; see [34].
}

custodial symmetry at finite temperature is checked to be negligible for the SFOEWPT parameter space under study (see Ref. [21] for details). In the one-step phase transition case, the phase transition occurs through the path of $A \rightarrow B$ directly with $h_{\phi, \xi}^{B}$ located at around $v_{\phi, \xi}$ at the finite temperature $T_{C}$. The phase transition may occur after the temperature drops below $T_{C}$. In Fig. 2, we illustrate the one-step phase transition process as the temperature drops. The global minimum of the finite temperature potential $V_{T}$ changes from $A$ to $B$, through which one obtains the EW symmetry breaking minimum.

As for the two-step phase transition scenario, the condition for the first step of the two-step phase transition (Apoint $\rightarrow C$ point) can be written as:

$$
\begin{aligned}
& V_{T}\left(0,0, T_{1 C}\right)=V_{T}\left(0, h_{\xi}^{1 C}, T_{1 C}\right), \\
& \left.\frac{d V_{T}\left(h_{\phi}, h_{\xi}, T_{1 C}\right)}{d h_{\xi}}\right|_{h_{\phi}=0, h_{\xi}=h_{\xi}^{1 C}}=0 .
\end{aligned}
$$

The critical parameters $h_{\xi}^{1 C}$ and $T_{1 C}$ are calculated as,

$h_{\xi}^{1 C}=-\frac{\mu_{2}}{3 \lambda_{2}+\lambda_{3}}, \quad T_{1 C}=\frac{\sqrt{-3 m_{2}^{2}\left(3 \lambda_{2}+\lambda_{3}\right)+6 \mu_{2}^{2}}}{\sqrt{\left(c_{\xi}+2 c_{\chi}\right)\left(3 \lambda_{2}+\lambda_{3}\right)}}$.

For the second-step of the two-step phase transition to occur, the following degeneracy conditions at the critical temperature are necessary:

$$
\begin{aligned}
V_{T}\left(0, h_{\xi}^{C}, T_{C}\right) & =V\left(h_{\phi}^{B}, h_{\xi}^{B}, T_{C}\right), \\
\left.\frac{d V_{T}\left(h_{\phi}, h_{\xi}, T_{C}\right)}{d h_{\phi}}\right|_{h_{\phi}=h_{\phi}^{B}, h_{\xi}=h_{\xi}^{B}} & =0, \\
\left.\frac{d V_{T}\left(h_{\phi}, h_{\xi}, T_{C}\right)}{d h_{\xi}}\right|_{h_{\phi}=h_{\phi}^{B}, h_{\xi}=h_{\xi}^{B}} & =0, \\
\left.\frac{d V_{T}\left(0, h_{\xi}, T_{C}\right)}{d h_{\xi}}\right|_{h_{\xi}=h_{\xi}^{C}} & =0,
\end{aligned}
$$


through which the critical temperature and critical field value can be obtained. For the two-step case, using the determinant of the Hessian matrix (at both the zero temperature and the finite temperature) to ensure that the two degenerate vacua occur, the following conditions need to be satisfied: $M_{3(5,6)} P_{3(5,6)}-N_{3(5,6)}^{2}>0, M_{3(5,6)}>0$, with

$$
\begin{aligned}
&\left.\frac{d^{2} V_{0}\left(h_{\phi}, h_{\xi}\right)}{d h_{\phi}^{2}}\right|_{h_{\phi}=v_{\phi}, h_{\xi}=v_{\xi}} \equiv M_{3},\left.\quad \frac{d^{2} V_{0}\left(h_{\phi}, h_{\xi}\right)}{d h_{\phi} d h_{\xi}}\right|_{h_{\phi}=v_{\phi}, h_{\xi}=v_{\xi}} \equiv N_{3}, \\
&\left.\frac{d^{2} V_{0}\left(h_{\phi}, h_{\xi}\right)}{d h_{\xi}^{2}}\right|_{h_{\phi}=v_{\phi}, h_{\xi}=v_{\xi}} \equiv P_{3}, \\
&\left.\frac{d^{2} V_{T}\left(h_{\phi}, h_{\xi}, T_{2 C}\right)}{d h_{\phi}^{2}}\right|_{h_{\phi}=h_{\phi}^{B}, h_{\xi}=h_{\xi}^{B}} \equiv M_{5},\left.\quad \frac{d^{2} V_{T}\left(h_{\phi}, h_{\xi}, T_{2 C}\right)}{d h_{\phi} d h_{\xi}}\right|_{h_{\phi}=h_{\phi}^{B}, h_{\xi}=h_{\xi}^{B}} \equiv \\
&\left.\frac{d^{2} V_{T}\left(h_{\phi}, h_{\xi}, T_{2 C}\right)}{d h_{\xi}^{2}}\right|_{h_{\phi}=h_{\phi}^{B}, h_{\xi}=h_{\xi}^{B}} \equiv P_{5}, \\
&\left.\frac{d^{2} V_{T}\left(h_{\phi}, h_{\xi}, T_{2 C}\right)}{d h_{\phi}^{2}}\right|_{h_{\phi}=0, h_{\xi}=h_{\xi}^{2 C}} \equiv M_{6},\left.\quad \frac{d^{2} V_{T}\left(h_{\phi}, h_{\xi}, T_{2 C}\right)}{d h_{\phi} d h_{\xi}}\right|_{h_{\phi}=0, h_{\xi}=h_{\xi}^{2 C}} \equiv \\
&\left.\frac{d^{2} V_{T}\left(h_{\phi}, h_{\xi}, T_{2 C}\right)}{d h_{\xi}^{2}}\right|_{h_{\phi}=0, h_{\xi}=h_{\xi}^{2 C}} \equiv P_{6} .
\end{aligned}
$$

Here, at finite temperature $T_{2 C}, h_{\phi, \xi}^{B}$ is located at around $v_{\phi, \xi}$, and $h_{\xi}^{2 C}$ at around $h_{\xi_{C_{1}(2)}}$ as given in Eq. (21). That the temperature of the first step phase transition is higher than the second one, i.e., $T_{1 C}>T_{2 C}$, is also used to select the SFOEWPT points for the two step phase transition scenario.

At the zero temperature, the positiveness of the Hessian matrix determinants can allow one local minimum at $\mathrm{C} 1$ or $\mathrm{C} 2$, or both $\mathrm{C} 1$ and $\mathrm{C} 2$, in addition to the global vacuum located at $B$. We show in Fig. 3 how the phase transitions occur as the temperature drops in the two-step case. During these two-step phase transition processes, the global vacuum of the finite temperature potential $V_{T}$ changes from $A$ to $C_{1,2}$ at the first-step after $T<T_{1 C}$. Then, the phase changes from the vacuum located at around $C_{1,2}$ to the one around $B$, i.e., the EW symmetry breaking vacuum, after $T<T_{2 C}$. The Hessian matrix at finite and zero temperature tells if there exist local or global minima at $C_{1}, C_{2}$, and $B$ at the corresponding temperature; see Eq. (33). In the BP1 (top panel of Fig. 3), there exists only the minima around $C_{2}$ at zero temperature and the finite temperature. In the BP2 (bottom panel of Fig. 3), only the $C_{2}$ exists at zero temperature, and both the $C_{1}$ and $C_{2}$ could exist at the second-step phase transition temperature of $T_{2 C}$.

The above procedure is firstly used to obtain the critical phase transition order parameters and the rough phase transition pattern (either one-step or two-step). Subsequently, we use CosmoTransitons [39] to obtain the phase transition order parameters at the bubble nucleation temperature, which might be slightly different from the patterns in the above approach. This is mainly due to the reason that in some cases, although we can obtain the phase transition with the above approach, the improper barrier between the two minima at the bubble nucleation temperature could not fulfill the condition of bubble nucleation; see Eq. (55). This is especially important for the two-step cases where the pattern would be changed from $A \rightarrow$ $C_{1,2} \rightarrow B$ to $A \rightarrow B$. Hence, when we present the results, the term "two-step" refers to the points obtained by the above approach, and the terms "bubble one-step" and "bubble two-step" refer to the points that can trigger one-step bubble nucleation and two-step bubble nucleation respectively after we check the bubble nucleation using CosmoTransitions.

Before concluding this section, we remind the reader that, the one-step phase transition as shown in Fig. 2 does not allow the homotopy group for the domain wall. The vacuum manifold for the second-step of the two-step phase transition as shown in Fig. 3 admits a nontrivial homotopy group $\pi_{0}\left(H_{3} / H_{2}\right)$ [with $H_{3}=Z_{2} \times S U(2)_{V}$ and $H_{2}=$ $S U(2)_{V}$ after the first-step and second-step phase transitions, respectively)], which allows the existence of domain wall [40] which can produce GW after decay through quantum tunneling [41]. We left detailed studies on topological defect and its GW signal after the SFOEWPT in GM model to the future study. 

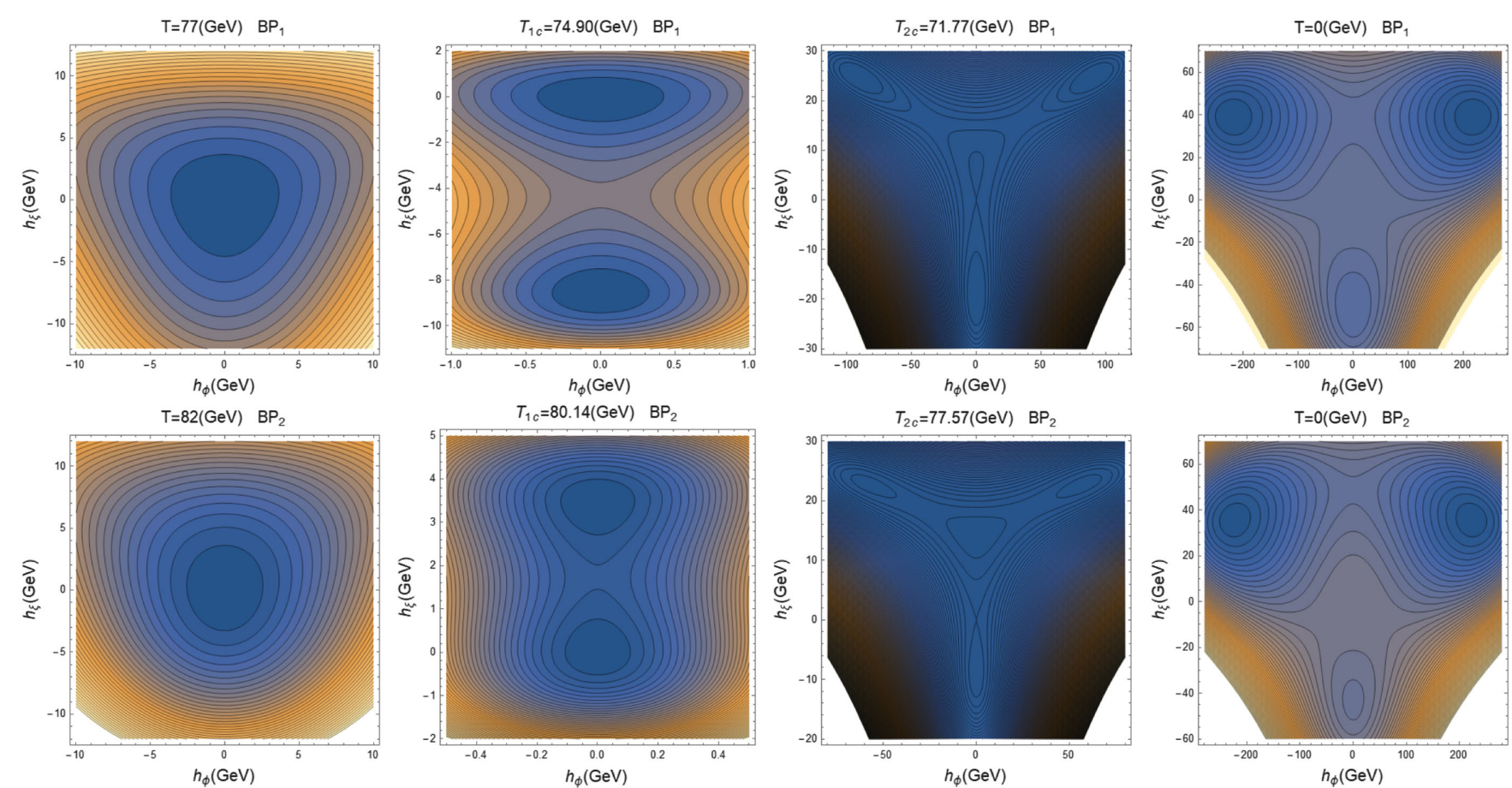

FIG. 3. The evolution of the vacuum structure as temperature drops. The evolution of the vacuum structure as temperature drops for the two-step SFOEWPT case. Upper Panels: $B P_{1}$ with the phase transition pattern of $A \rightarrow C_{2} \rightarrow B$. Lower Panels: $B P_{2}$ with the phase transition pattern of $A \rightarrow C_{1} \rightarrow B$. The parameters for $B P_{1}$ are: $\lambda_{1}=0.040, \lambda_{2}=0.598, \lambda_{3}=-0.113, \lambda_{4}=0.425, \lambda_{5}=0.264$, $\theta_{H}=0.467, \mu_{1}=-175.619 \mathrm{GeV}, \mu_{2}=14.416 \mathrm{GeV}$. The parameters for $B P_{2}$ are: $\lambda_{1}=0.039, \lambda_{2}=0.692, \lambda_{3}=-0.311, \lambda_{4}=0.479$, $\lambda_{5}=0.457, \theta_{H}=0.423, \mu_{1}=-181.111 \mathrm{GeV}, \mu_{2}=-6.144 \mathrm{GeV}$.

\section{THE SFOEWPT AND HIGGS PHENOMENOLOGY}

The vacuum structure at zero temperature, such as the potential barrier, is crucial for both one-step and two-step SFOEWPT. The desired vacuum structure for the SFOEWPT reveals the Higgs potential shape through the deviations of the triple and quartic Higgs couplings from the $\mathrm{SM}$ values. The typical vacuum structure for the one-step and two-step SFOEWPT being explored in the last section can have distinct Higgs phenomenological predictions.

In the GM model, both the two extra triplets contribute to the EWSB, and the gauge bosons get masses also from the triplet VEV, $v_{\xi, \chi}$. The electroweak charge of the triplets leads to the $H V V$ couplings deviating from the singlet case by one extra factor of $\sin \theta_{H}$, which parametrizes the contribution of the $v_{\xi, \chi}$ to the Higgs VEV, $\sin \theta_{H}=2 \sqrt{2} \nu_{\xi} / \sqrt{\left(8 v_{\xi}^{2}+v_{\phi}^{2}\right)}$ [31]. For the GM model, due to the contribution of the isospin triplet to the EWSB, the phase transition strength is defined as [21]

$$
\begin{aligned}
v^{G M} / T & \equiv \frac{\sqrt{v_{\phi}^{2}(T)+8 v_{\xi}^{2}(T)}}{T}=\frac{v_{\phi}(T) \cos \theta_{H}(T)^{-1}}{T}, \\
\cos \theta_{H}(T) & \equiv \frac{v_{\phi}(T)}{\sqrt{v_{\phi}^{2}(T)+8 v_{\xi}^{2}(T)}},
\end{aligned}
$$

at the critical temperature when the phase transition occurs. Since we are working in the scenario where the zero temperature vacuum structure is crucial for the SFOEWPT, the $\theta_{H}(T)$ here would be highly related with $\theta_{H}$ (see our previous studies in Ref. [21] for details). As will be explored latter, one can expect that the one-step and two-step SFOEWPT valid regions are highly restricted by collider searches.

To study the collider phenomenology of the new physics models, one needs to work in the physical eigenbasis. In terms of physical field basis after taking into account of the rotation matrix (with the angle $\alpha_{H}$ ) among classical fields and Higgs fields $h, H$, the interaction strength between the SM-like Higgs and SM particles are:

$$
\begin{aligned}
g_{h f \bar{f}} & =\cos \alpha_{H} / \cos \theta_{H} g_{h f \bar{f}}^{\mathrm{SM}}, \\
g_{h V V} & =\left(\cos \alpha_{H} \cos \theta_{H}-\sqrt{\frac{8}{3}} \sin \alpha_{H} \sin \theta_{H}\right) g_{h f \bar{f}}^{\mathrm{SM}}, \\
g_{H f \bar{f}} & =\sin \alpha_{H} / \cos \theta_{H} g_{h f \bar{f}}^{\mathrm{SM}}, \\
g_{H V V} & =\left(\sin \alpha_{H} \cos \theta_{H}+\sqrt{\frac{8}{3}} \cos \alpha_{H} \sin \theta_{H}\right) g_{h V V}^{\mathrm{SM}} .
\end{aligned}
$$

Currently, the angle $\theta_{H}$ as a function of doubly charged Higgs boson mass is severely bounded by the same-sign 
$W W$ boson channel searches at $13 \mathrm{TeV}$ LHC [27]. We adopt the observed 95\% CL upper limits given in Fig. 4 of Ref. [27] to bound the SFOEWPT parameter spaces. Future hadron and lepton colliders would further restrict the magnitude of $\alpha_{H}$ and $\theta_{H}$, which means that the possibility to reach SFOEWPT would be confined to the parameter space with small $\sin \theta_{H}$ and $\alpha_{H}$. For the case of the small $\theta_{H}$ limit where the EWSB contribution from the triplet is negligible, one will also have

$$
\alpha_{H} \approx-\sqrt{\frac{3}{2}} \theta_{H}+\frac{\theta_{H}^{2}\left(8 \sqrt{3} \lambda_{1}-2 \sqrt{3} \lambda_{4}-\sqrt{3} \lambda_{5}\right) \nu}{\mu_{1}} .
$$

This means that, for a small $\theta_{H}$, one usually has a small $\alpha$, and the sign of $\alpha_{H}$ is determined by the combination of couplings $8 \sqrt{3} \lambda_{1}-2 \sqrt{3} \lambda_{4}-\sqrt{3} \lambda_{5}$ and $\mu_{1}$. In the scenario with small $\alpha$ and small $\theta_{H}, g_{h f \bar{f}, h V V}$ is close to the SM case, while $g_{H f \bar{f}, H V V}$ is suppressed.

The scalar potential of Eq. (5) in the Higgs basis of $h$ and $H$ can be written as,

$$
\begin{aligned}
V_{\mathrm{phy}}^{\mathrm{GM}}= & \frac{1}{2} m_{1}^{2}\left(h \cos \alpha_{H}+H \sin \alpha_{H}\right)^{2}+\frac{1}{2} m_{2}^{2}\left(H \cos \alpha_{H}-h \sin \alpha_{H}\right)^{2} \\
& +\frac{2}{\sqrt{3}} \mu_{2}\left(H \cos \alpha_{H}-h \sin \alpha_{H}\right)^{3}+\frac{\sqrt{3}}{4} \mu_{1}\left(h \cos \alpha_{H}+H \sin \alpha_{H}\right)^{2}\left(H \cos \alpha_{H}-h \sin \alpha_{H}\right) \\
& +\lambda_{1}\left(h \cos \alpha_{H}+H \sin \alpha_{H}\right)^{4}+\left(\lambda_{2}+\frac{1}{3} \lambda_{3}\right)\left(H \cos \alpha_{H}-h \sin \alpha_{H}\right)^{4} \\
& +\left(\lambda_{4}+\frac{1}{2} \lambda_{5}\right)\left(h \cos \alpha_{H}+H \sin \alpha_{H}\right)^{2}\left(H \cos \alpha_{H}-h \sin \alpha_{H}\right)^{2}
\end{aligned}
$$

for the GM model. In the small $\alpha_{H}$ limit, the potential $V_{\text {phy }}^{\mathrm{GM}}$ reduces to

$$
\begin{aligned}
V_{\alpha}^{\mathrm{GM}}= & \frac{1}{2} m_{1}^{2} h^{2}+\lambda_{1} h^{4}+\frac{1}{2} m_{2}^{2} H^{2}+\frac{2 \sqrt{3}}{3} \mu_{2} H^{3}+\frac{1}{3}\left(3 \lambda_{2}+\lambda_{3}\right) H^{4}+\frac{\sqrt{3}}{4} \mu_{1} h^{2} H \\
& +\frac{1}{2}\left(2 \lambda_{4}+\lambda_{5}\right) h^{2} H^{2}+\alpha_{H} h\left(-\frac{\sqrt{3} \mu_{1}}{4} h^{2}+\left(m_{1}^{2}-m_{2}^{2}\right) H+\frac{\sqrt{3}}{2}\left(\mu_{1}-4 \mu_{2}\right) H^{2}\right. \\
& \left.+\left(-4 \lambda_{2}-\frac{4 \lambda_{3}}{3}+2 \lambda_{4}+\lambda_{5}\right) H^{3}+\left(4 \lambda_{1}-2 \lambda_{4}-\lambda_{5}\right) h^{2} H\right)+\mathcal{O}\left(\alpha_{H}^{2}\right) .
\end{aligned}
$$

After EWSB, $h$ and $H$ get VEVs,

$$
\begin{aligned}
& v_{h}^{\mathrm{GM}}=v \cos \left(\alpha_{H}\right) \cos \left(\theta_{H}\right)-\frac{1}{2} \sqrt{\frac{3}{2}} v \sin \left(\alpha_{H}\right) \sin \left(\theta_{H}\right), \\
& v_{H}^{\mathrm{GM}}=v \sin \left(\alpha_{H}\right) \cos \left(\theta_{H}\right)+\frac{1}{2} \sqrt{\frac{3}{2}} v \cos \left(\alpha_{H}\right) \sin \left(\theta_{H}\right),
\end{aligned}
$$

with $v=v_{\mathrm{SM}} \equiv 246 \mathrm{GeV}$. Suppose $h$ is the SM-like Higgs, one has both the Higgs cubic and quartic couplings modified compared with the SM case. This can be parametrized as:

$$
\Delta \mathcal{L}=-\frac{1}{2} \frac{m_{h}^{2}}{v}\left(1+\delta \kappa_{3}\right) h^{3}-\frac{1}{8} \frac{m_{h}^{2}}{v^{2}}\left(1+\delta \kappa_{4}\right) h^{4} .
$$

The cubic Higgs couplings are crucial for the vacuum structure, and therefore the phase transition dynamics, as well as the Higgs pair production at hadron and lepton colliders. Consequently, the Higgs pair searches can be powerful enough to probe the parameter space of the SFOEWPT. In the small $\alpha\left(\theta_{H}\right)$ limit, we have

$$
\begin{gathered}
\delta \kappa_{3}^{G M}=-\alpha_{H} \frac{\sqrt{3} \mu_{1} v}{2 m_{h}^{2}}+\frac{\alpha_{H} v^{2}\left(4 \alpha_{H}-\sqrt{6} \theta_{H}\right)\left(2 \lambda_{4}+\lambda_{5}\right)}{2 m_{h}^{2}} \\
-\frac{\left(3 \alpha_{H}^{2}+\theta_{H}^{2}\right)}{2}+\mathcal{O}\left(\alpha_{H}^{3}, \theta_{H}^{3}\right), \\
\delta \kappa_{4}^{G M}=-2 \alpha_{H}^{2}\left(1-\frac{2\left(2 \lambda_{4}+\lambda_{5}\right) v^{2}}{m_{h}^{2}}\right)+\mathcal{O}\left(\alpha_{H}^{3}\right) .
\end{gathered}
$$

As a comparison, we also list the $\mathrm{xSM}$ case which has no extra EWSB contribution. In the xSM case, we also define a mixing angle $\alpha_{H}$ between the SM Higgs $(h)$ and extra scalar $(s)$. At the zero temperature, it is defined as [38]

$$
\sin 2 \alpha_{H}=\frac{\left(a_{1}+2 a_{2} v_{s}\right) v_{h}}{\left(m_{h}^{2}-m_{H}^{2}\right)}
$$


with $v_{h}=246 \mathrm{GeV}$. For $-1 \leq \sin 2 \alpha_{H} \leq 1$, this sets a bound on the VEV fraction of the SM Higgs $(h)$,

$$
m_{h}^{2}-m_{H}^{2} \leq\left(a_{1}+2 a_{2} v_{s}\right) v_{h} \leq m_{H}^{2}-m_{h}^{2} .
$$

The phase transition occurs in the subspace of the two scalar fields, with the phase transition strength being

$$
\begin{aligned}
v^{\mathrm{xSM}} / T & \equiv \frac{v_{h}(T)}{T}=\frac{\sqrt{v_{h}^{2}(T)+v_{s}^{2}(T)} \cos \theta(T)}{T}, \\
\cos \theta(T) & \equiv \frac{v_{h}(T)}{\sqrt{v_{h}^{2}(T)+v_{s}^{2}(T)}} .
\end{aligned}
$$

The sphaleron process is quenched when $v_{h}(T) / T>1$ at the critical temperature. Different from the GM model, the singlet $\operatorname{VEV}\left(v_{s}\right)$ does not contribute to the mass of gauge boson due to the electroweak charge of the singlet, hence it does not contribute to the phase transition strength.

The collider phenomenology of the Higgs searches is based on the measurements of the SM Higgs and searches for an extra heavy Higgs-like scalar. The current LHC Higgs data and theoretical constraints require small $\alpha_{H}$ which parametrizes the mixing between the SM-like Higgs and the extra $C P$-even heavy Higgs. In this model, all the couplings are rescaled by $\alpha_{H}$ based on the SM as: $g_{h x x}=\cos \alpha_{H} g_{h x x}^{\mathrm{SM}}, g_{H x x}=-\sin \alpha_{H} g_{h x x}^{\mathrm{SM}}$. Therefore, no direct bound on the angle $\theta(T=0)$ from the Higgs data since the parameter does not enter Higgs couplings. The VEV of the extra scalar $\left(v_{s}\right)$ would be more free than that in the GM model.

For the $\mathrm{xSM}$ model, the potential in the basis of Higgs fields is given by

$$
\begin{aligned}
V_{\text {phy }}^{\mathrm{xSM}}= & \frac{1}{12}\left(3 a_{1}\left(h \cos \alpha_{H}-H \sin \alpha_{H}\right)^{2}\left(h \sin \alpha_{H}+H \cos \alpha_{H}\right)+3 a_{2}\left(h \cos \alpha_{H}-H \sin \alpha_{H}\right)^{2}\right. \\
& \times\left(h \sin \alpha_{H}+H \cos \alpha_{H}\right)^{2}+6 b_{2}\left(h \sin \alpha_{H}+H \cos \alpha_{H}\right)^{2}+4 b_{3}\left(h \sin \alpha_{H}+H \cos \alpha_{H}\right)^{3} \\
& \left.+3 b_{4}\left(h \sin \alpha_{H}+H \cos \alpha_{H}\right)^{4}+3 \lambda\left(h \cos \alpha_{H}-H \sin \alpha_{H}\right)^{4}-6 \mu^{2}\left(h \cos \alpha_{H}-H \sin \alpha_{H}\right)^{2}\right) .
\end{aligned}
$$

Going to the alignment case, one has

$$
\begin{aligned}
V_{\alpha}^{\mathrm{xSM}}= & \frac{1}{12}\left(3 a_{1} h^{2} H+3 a_{2} h^{2} H^{2}+6 b_{2} H^{2}+4 b_{3} H^{3}+3 b_{4} H^{4}+3 h^{4} \lambda-6 h^{2} \mu^{2}\right) \\
& +\frac{1}{4} h \alpha_{H}\left(a_{1}\left(h^{2}-2 H^{2}\right)+2 H\left(a_{2}\left(h^{2}-H^{2}\right)+2 b_{2}+2\left(H\left(b_{3}+b_{4} H\right)-\lambda h^{2}+\mu^{2}\right)\right)\right)+\mathcal{O}\left(\alpha_{H}^{2}\right) .
\end{aligned}
$$

After EWSB, one has for the two physical fields $h$ and $H$ :

$$
\begin{aligned}
& v_{h}^{\mathrm{xSM}}=v_{h} \cos \left(\alpha_{H}\right)+v_{s} \sin \left(\alpha_{H}\right), \\
& v_{H}^{\mathrm{xSM}}=v_{s} \cos \left(\alpha_{H}\right)-v_{h} \sin \left(\alpha_{H}\right) .
\end{aligned}
$$

In the $\mathrm{xSM}$, the deviations of the cubic and quartic couplings for small $\alpha_{H}$ are given by [42]

$$
\begin{aligned}
& \delta \kappa_{3}^{\mathrm{xSM}}=\alpha_{H}^{2}\left[-\frac{3}{2}+\frac{2 m_{H}^{2}-2 b_{3} v_{s}-4 b_{4} v_{s}^{2}}{m_{h}^{2}}\right]+\mathcal{O}\left(\alpha_{H}^{3}\right), \\
& \delta \kappa_{4}^{\mathrm{xSM}}=\alpha_{H}^{2}\left[-3+\frac{5 m_{H}^{2}-4 b_{3} v_{s}-8 b_{4} v_{s}^{2}}{m_{h}^{2}}\right]+\mathcal{O}\left(\alpha_{H}^{3}\right) .
\end{aligned}
$$

Due to the extra EWSB contribution, in the GM model, one has an additional angle $\theta_{H}$ to parametrize the Higgs couplings. Therefore one has the different distributions of SFOEWPT points in the $v_{\xi}-\alpha_{H}$ plane, which builds the bridge between the SFOEWPT and the Higgs phenomenology. Figure 4 shows the one-step (red) and two-step (blue) SFOEWPT valid points in the xSM (left) and GM (right) model. For the xSM case, the one-step (two-step)
SFOEWPT points are concentrated in the small (large) $v_{s}$ regions. While in the GM model, $v_{\xi}$ is much smaller than $v_{s}$ of the XSM and the possibility to reach a SFOEWPT drops as $v_{\xi}$ and $\left|\sin \alpha_{H}\right|$ decrease.

In the parameter space which allows the SFOEWPT, one has the deviations of the cubic and quartic Higgs couplings, which characterize the Higgs potential shapes that are crucial for the realization of the EWSB mechanism. We investigate more details in two particular benchmarks of the GM model: the H5plane which is developed by the LHC Higgs Cross Section Working Group for fiveplet searches [43] and the low mass benchmark studied in [44] for the lower mass region. Both benchmark scenarios will lead to interesting searches at the collider. As we have stated above, the current strongest constraint is from the samesign $W$ pair searches from CMS [27]. We implemented this constraint during our parameter scans. The vector boson fusion (VBF) production cross section of the double charged scalar is calculated using MadGraph [45] while the decay branching ratio is gotten from GMCalc [32]. Then at each mass point within the range that the experiments explored, the total cross section should be less than 

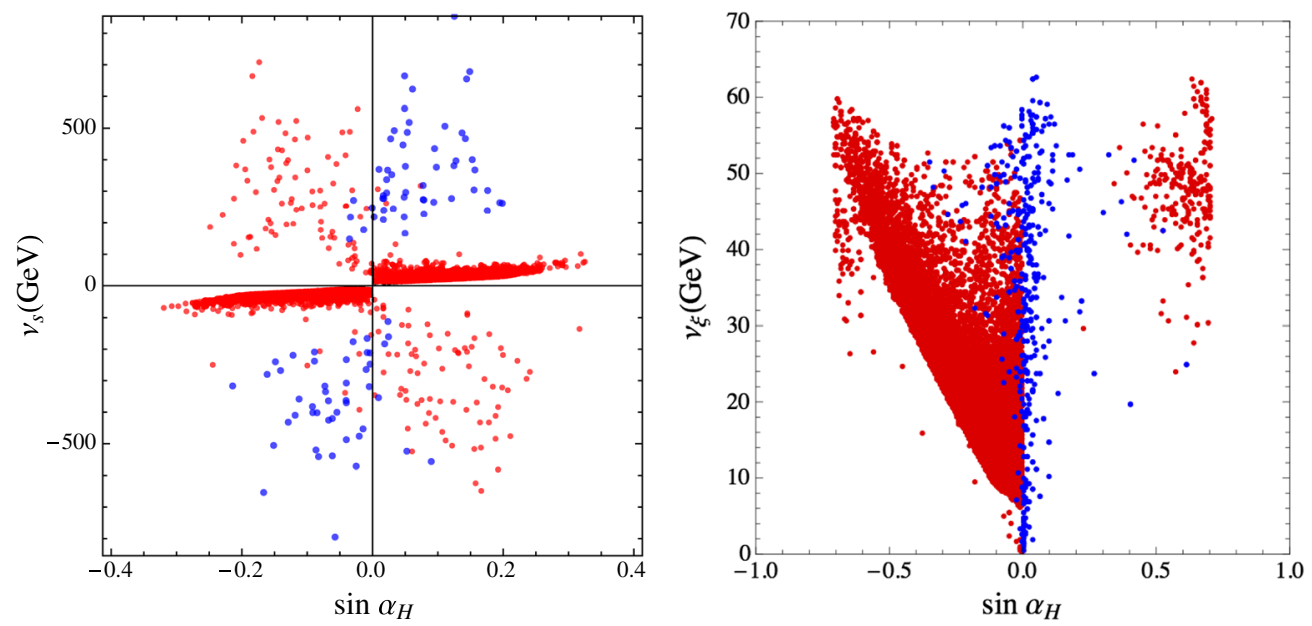

FIG. 4. The $v_{C} / T_{C}>1$ viable points [both one-step (red) and two-step (blue)] in the $\sin \alpha_{H}-v_{s, \xi}$ plane for the xSM (left) and the GM (right) model.
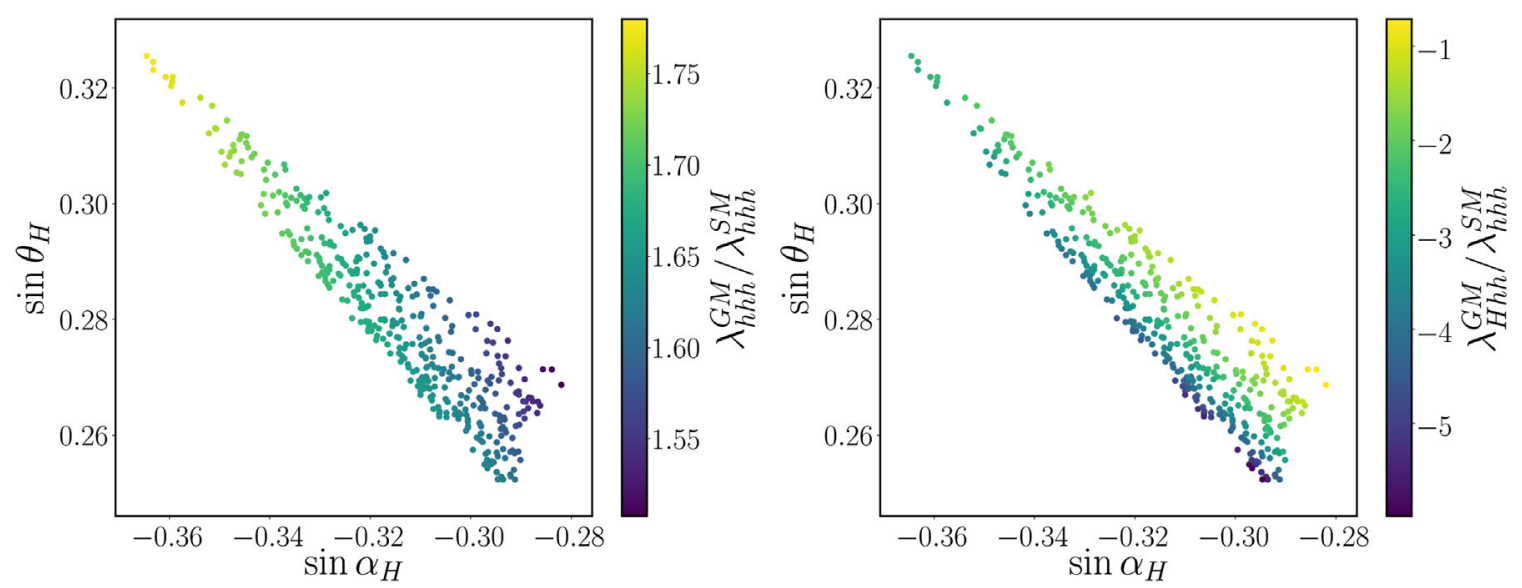

FIG. 5. The ratio of $\lambda_{h h h}^{\mathrm{GM}} / \lambda_{h h h}^{\mathrm{SM}}$ (left) and $\lambda_{H h h}^{\mathrm{GM}} / \lambda_{h h h}^{\mathrm{SM}}$ (right) in the $\sin \alpha_{H}-\sin \theta_{H}$ plane in the case of H5plane.

the upper limits in [27]. Note that the sensitivity of the same-sign $W$ pair searches only starts from $200 \mathrm{GeV}$. Hence, without any extrapolation, although the constraint on $\sin \theta_{H}$ at large mass is quite stringent, for the mass below $200 \mathrm{GeV}$, larger $\sin \theta_{H}$ is still allowed.

For the physical intuition of the phase transition, in comparison with the H5plane, the main feature of the parameter space of the low mass benchmark is the relatively lower magnitude of the fiveplet mass and the smallness of the angles of $\theta_{H}$ and $\alpha_{H}$, which make the two-step SFOEWPT possible. As can be found in Ref. [21], the one-step SFOEWPT mostly occurs with a negative mixing angle $\alpha_{H}$, a larger magnitude of $\left|\mu_{1,2}\right|$, and favors a relatively large $m_{h_{5}}, m_{h_{3}}$, and $m_{H}$ in comparison with the two-step SFOEWPT.

Our previous study in [21] shows that only one-step SFOEWPT is valid in H5plane, while the low mass benchmark can provide both one-step and two-step SFOEWPT. After considering the current LHC search bounds, especially the same-sign $W$ search from CMS [27], we show the triple scalar couplings in Figs. 5 and 6 for the H5plane and low mass benchmark, respectively. In H5plane, Fig. 5 shows that a larger deviation of the triple Higgs coupling $\lambda_{h h h}^{\mathrm{GM}}$ from the SM one occurs with a higher magnitude of $\left|\alpha_{H}\right|$ and a larger $\theta_{H}$. While the $\lambda_{H h h}^{\mathrm{GM}}$ is highly enhanced with an extra sign compared with $g_{h h h}^{\mathrm{SM}}$ which can result in destructive interference for Higgs pair production at the colliders.

In low mass benchmark, one has smaller $\sin \theta_{H}$ and $m_{h_{5}}$ in comparison with the H5plane scenario. The CMS same$\operatorname{sign} W$ search severely bounds $\sin \theta_{H}$ and thus the mixing angle $\alpha_{H}$. Therefore one can expect that the triple Higgs coupling and the quartic Higgs couplings are all restricted. In Fig. 6, we show the triple Higgs couplings in the $\sin \alpha_{H}-\sin \theta_{H}$ plane for both one (upper panels) and twostep (lower panels) SFOEWPT. The SFOEWPT viable points in low mass benchmark are located at smaller values of $\theta_{H}$ and $\alpha_{H}$. Thus we have smaller enhancement in $\lambda_{h h h}^{\mathrm{GM}}$ 

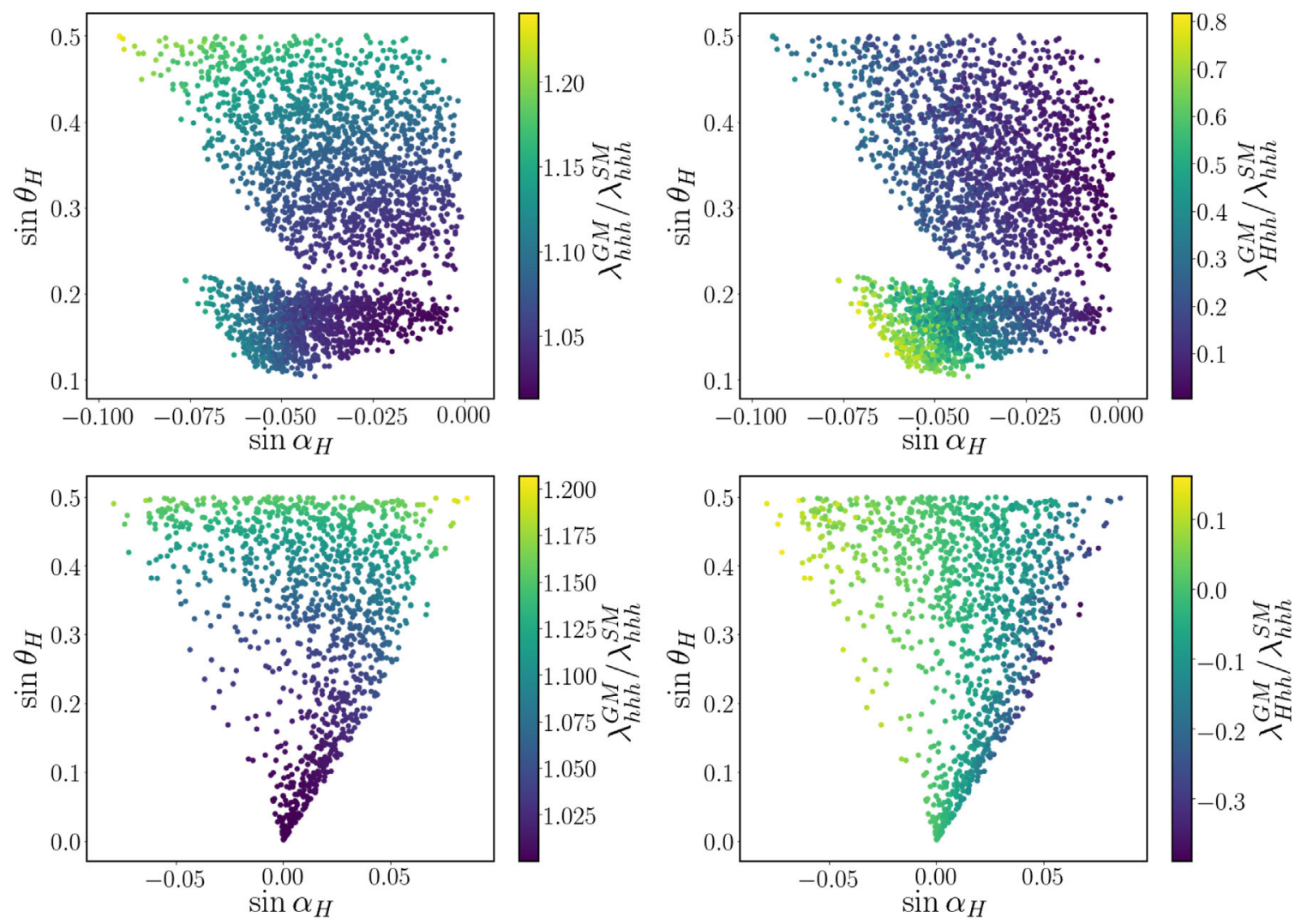

FIG. 6. The ratio of $\lambda_{h h h}^{\mathrm{GM}} / \lambda_{h h h}^{\mathrm{SM}}$ (left panels) and $\lambda_{H h h}^{\mathrm{GM}} / \lambda_{h h h}^{\mathrm{SM}}$ (right panels) in the $\sin \alpha_{H}-\sin \theta_{H}$ plane for both one-step points (upper panels) and two-step points (lower panels) in the case of the low mass benchmark.

than that in the H5plane. Meanwhile, $\lambda_{H h h}^{\mathrm{GM}}$ is also much smaller than that in the H5plane. These will result in different gravitational wave production and collider phenomenology as we will study in the following.

A SFOEWPT can also be reached with the help of the dimensional six operator $\left(H^{\dagger} H\right)^{3}[46,47]$ or new physics that can contribute to such an operator. The collider could not tell the detailed potential shape (i.e., the tree level potential barrier) that drives the phase transition. In this case, the gravitational wave searches of the signal generated by the SFOEWPT would be complementary, since it captures the tunneling process manifested in terms of the vacuum bubble nucleations [9].

\section{GRAVITATIONAL WAVE SEARCHES}

When the temperature of the Universe further cools down after the critical temperature $T_{C}$ (where one has the degeneracy of the true and the false vacuum), one may have vacuum bubble nucleations, expansions, and collisions, and therefore GW production from the SFOEWPT process.

The bounce configuration of the nucleated bubble [the bounce configuration of the multifields that connects the EW broken vacuum ( $h$-vacuum, the true vacuum located around the $\mathrm{B}$ point) and the false vacuum (the vacuum locates around the $\mathrm{A}$ or $\mathrm{C}$ points)] can be obtained by extremizing

$$
S_{3}(T)=\int 4 \pi r^{2} d r\left[\frac{1}{2}\left(\frac{d \phi_{b}}{d r}\right)^{2}+V\left(\phi_{b}, T\right)\right],
$$

through solving the equation of motion for $\phi_{b}$ (it is $h$ and $h_{\xi}$ for two-step scenarios),

$$
\frac{d^{2} \phi_{b}}{d r^{2}}+\frac{2}{r} \frac{d \phi_{b}}{d r}-\frac{\partial V\left(\phi_{b}\right)}{\partial \phi_{b}}=0
$$

with the boundary conditions of

$$
\lim _{r \rightarrow \infty} \phi_{b}=0,\left.\quad \frac{d \phi_{b}}{d r}\right|_{r=0}=0 .
$$

The phase transition is completed approximately at the nucleation temperature when the thermal tunneling probability for bubble nucleation per horizon volume and per horizon time is of order unity [48-50]:

$$
\Gamma \approx A(T) e^{-S_{3} / T} \sim 1
$$

One of the crucial parameters for the gravitational wave spectra is $\alpha$, which is the energy density released from the SFOEWPT normalized by the total radiation energy density, defined as 

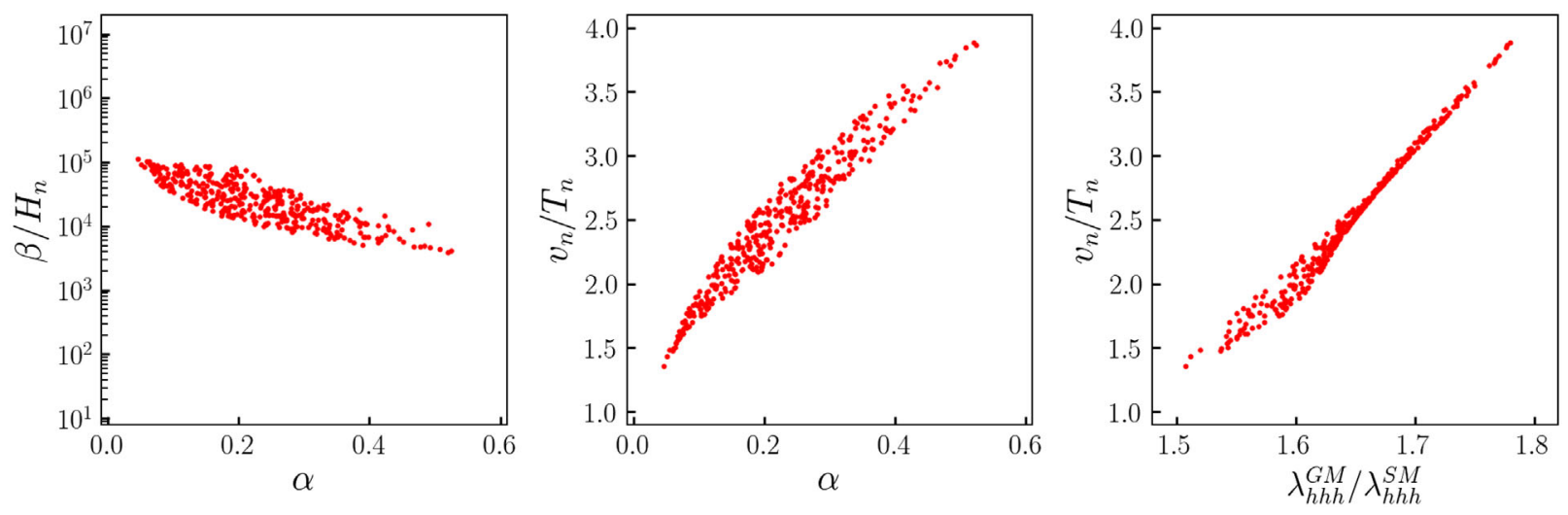

FIG. 7. The scan result in the $\alpha-\beta / H_{n}$ (left), $\alpha-v_{n} / T_{n}$ (middle), and $\lambda_{h h h}^{\mathrm{GM}} / \lambda_{h h h}^{\mathrm{SM}}-v_{n} / T_{n}$ (right) planes for the case of $H 5$ plane where we have only one-step (red) points.

$$
\alpha=\frac{\Delta \rho}{\rho_{R}},
$$

where the radiation energy density of the bath or the plasma background $\rho_{R}$ is given by

$$
\rho_{R}=\frac{\pi^{2} g_{\star} T_{\star}^{4}}{30}
$$

with $g_{\star}$ being the effective number of degrees of freedom (d.o.f.) $\left(g_{\star} \approx 100\right)$ at the plasma temperature $T_{\star}$ (when the phase transition is finished), which is approximately equivalent to the nucleation temperature $T_{\star} \approx T_{n}$ for transitions without significant reheating [51]. The parameter $\Delta \rho$ is the latent heat from the phase transition. This is given by the difference of the energy density between the false [here it is $\phi$ vacuum, $\left.\rho\left(\phi_{n}, T\right)\right]$ and the true vacuum [the $h$-vacuum or EW broken vacuum, $\rho\left(v_{n}, T\right)$ ], i.e., $\Delta \rho=\rho\left(\phi_{n}, T_{n}\right)-\rho\left(v_{n}, T_{n}\right)$, and ${ }^{3}$

$$
\begin{gathered}
\rho\left(\phi_{n}, T_{n}\right)=-\left.V(\phi, T)\right|_{T=T_{n}}+\left.T \frac{d V(\phi, T)}{d T}\right|_{T=T_{n}}, \\
\rho\left(v_{n}, T_{n}\right)=-\left.V(h, T)\right|_{T=T_{n}}+\left.T \frac{d V(h, T)}{d T}\right|_{T=T_{n}} .
\end{gathered}
$$

Here, we remind the reader that $\alpha$ and $\rho$ in this section represent the latent heat and energy density, rather than the mixing angle and electroweak parameter used in previous sections. Another crucial parameter $\beta$, which characterizes the inverse time duration of the SFOEWPT and thus the GW spectrum peak frequency, is defined as

\footnotetext{
${ }^{3}$ In our calculation, we use the latent heat by including the entropy injection from the phase transition (through the term of $\left.T \frac{d V}{d T}\right|_{T=T_{n}}$ ) as in Refs. [52-55] and some other literature, which coincides with the vacuum energy for the large supercooling phase transition case, as commented on in Ref. [51].
}

$$
\frac{\beta}{H_{n}}=\left.T \frac{d\left(S_{3}(T) / T\right)}{d T}\right|_{T=T_{n}},
$$

with $H_{n}$ the Hubble constant at the bubble nucleation temperature $T_{n}$.

Considering all the constraints from the LHC, especially the same-sign $W$ bounds from CMS [27], we show the results in Figs. 7 and 8 for the H5plane and the low mass benchmark respectively, where the red points represent the one-step scenario and the blue points represent the two-step case. As mentioned before, we separate the two-step points into two groups according to the scan results from CosmoTransition. The dark blue circles represent the case where we have two-step bubble nucleation ("bubble twostep"), ${ }^{4}$ while the light blue triangle points are those we have only one bubble nucleation ("bubble one-step").

In either the H5plane or the low mass benchmark (onestep and two-step), $\beta / H_{n}$ decreases as $\alpha$ increases. While $\alpha$ is also found to be proportional to the phase transition strength $v_{n} / T_{n}$, a larger value of $\alpha$ is obtained at a larger value of $v_{n} / T_{n}$ and results in a relatively larger energy density of the gravitational wave spectrum. As a comparison, besides the triple/quartic scalar coupling being smaller in the low mass benchmark, the low mass benchmark also provides lower $\beta / H_{n}$ in the similar range of $\alpha$ than that in the H5plane, which is necessary to produce detectable gravitational waves. In the low mass benchmark case, where we have both one-step and two-step scenarios, two-step SFOEWPT will have relatively smaller $\beta / H_{n}$ and larger $v_{n} / T_{n}$ for the same $\alpha$ than the one-step scenario.

Here the most stringent constraint comes from the CMS same-sign diboson search [27], which, however, does not extend to the mass $\left(m_{5}\right)$ below $200 \mathrm{GeV}$. This is not relevant for the H5plane, since all those points in Fig. 7 have $m_{5}>200 \mathrm{GeV}$. However, in the low mass benchmark, we

\footnotetext{
${ }^{4}$ The points correspond to the second step $\left(C_{1,2} \rightarrow B\right)$, while the first step $A \rightarrow C_{1,2}$ is not shown here which will be discussed later.
} 

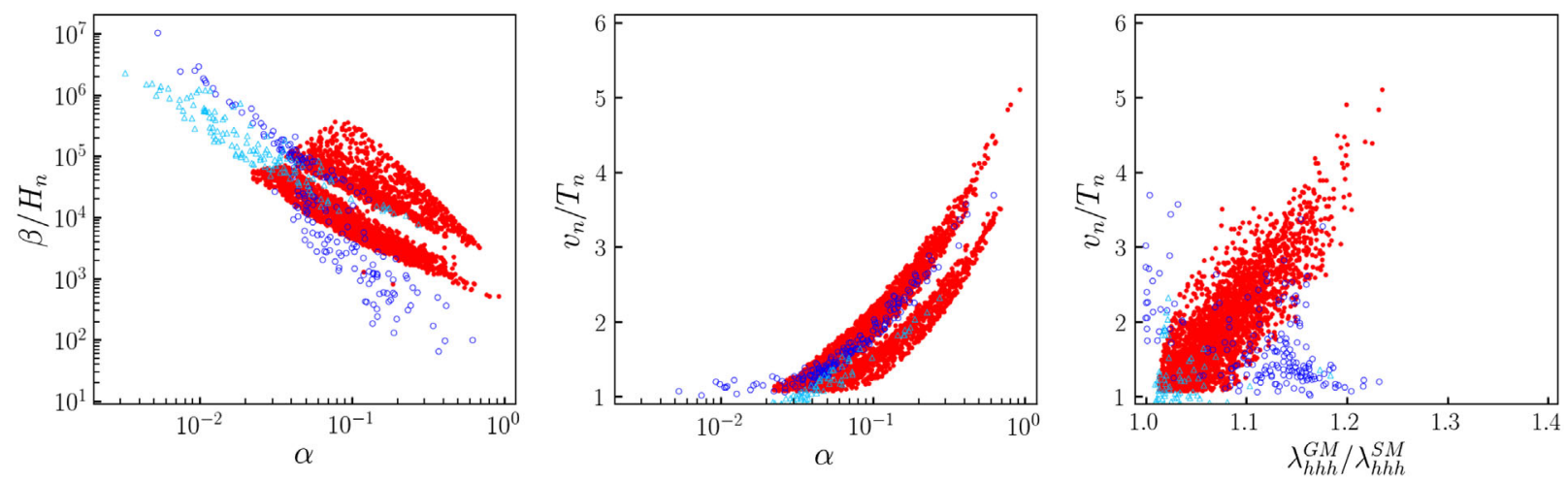

FIG. 8. The scan result in the $\alpha-\beta / H_{n}$ (left), $\alpha-v_{n} / T_{n}$ (middle), and $\lambda_{h h h}^{\mathrm{GM}} / \lambda_{h h h}^{\mathrm{SM}}-v_{n} / T_{n}$ (right) planes for the case of low mass benchmark where we have both one-step (red) and two-step (blue) points. Among the blue points, the circles represent the case where we can have two-step bubble nucleations ("bubble two-step"), while the triangle points are those where we have only one-step bubble nucleations ("bubble one-step").
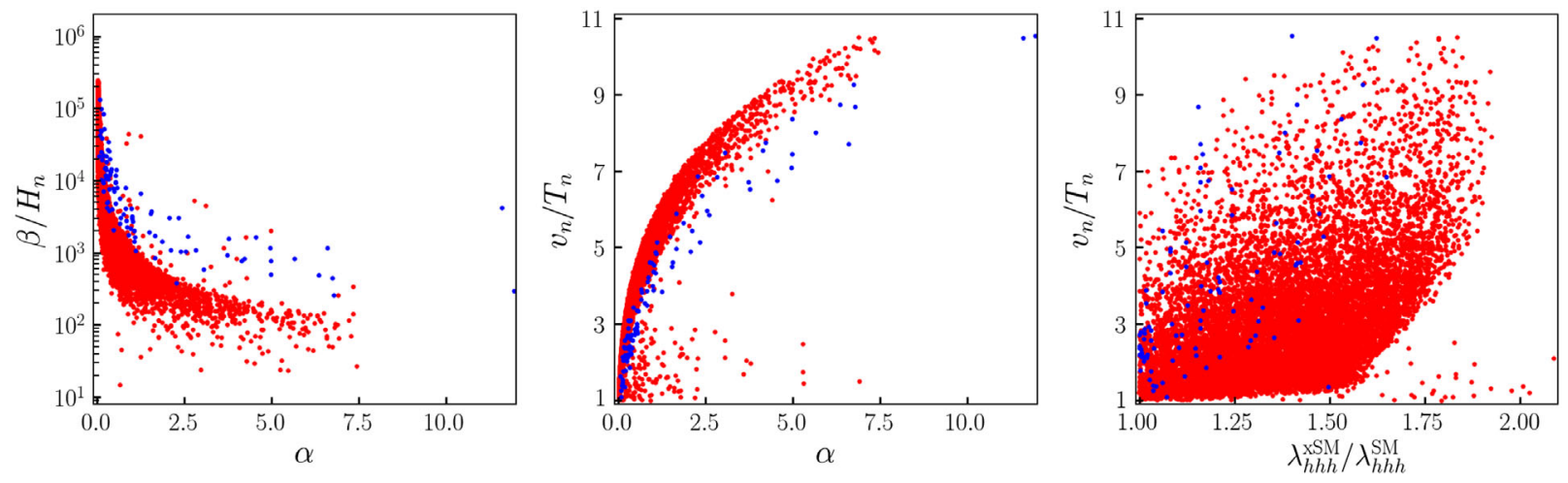

FIG. 9. SFOEWPT valid points in the $\alpha-\beta / H_{n}$ (left), $\alpha-v_{n} / T_{n}$ (middle), and $\lambda_{h h h}^{\mathrm{xSM}} / \lambda_{h h h}^{\mathrm{SM}}-v_{n} / T_{n}$ (right) planes for the xSM. Red and blue points represent the one- and two-step scenario, respectively.

can clearly see two parts of points for one-step scenario in Fig. 8. ${ }^{5}$ Those with lower $\beta / H_{n}$ or larger $v_{n} / T_{n}$ and thus more detectable GW signals, are the points having mass below $200 \mathrm{GeV}$, which require more dedicated searches at colliders. The triple Higgs couplings are also shown with respect to the SM scenario. The phase transition strength $v_{n} / T_{n}$ for one-step points is proportional to the triple Higgs coupling in both the H5plane and low mass benchmark, while there is no clear relation for the two-step points in the low mass benchmark.

At last, we comment on the situation in the xSM. In Fig. 9, we show the relations among $\beta / H_{n}, \alpha, v_{n} / T_{n}$, and triple scalar couplings in the $\mathrm{xSM}^{6}{ }^{6}$ Since the singlet VEV $v_{s}$ faces no restrictions in general in the $\mathrm{xSM}$, the magnitude of $\beta / H_{n}\left(v_{n} / T_{n}\right.$ and $\left.\alpha\right)$ is relatively much

\footnotetext{
${ }^{5}$ We do have two parts for two-step as well. However, the points with mass above $200 \mathrm{GeV}$ are the minority; we do not discuss them separately.

${ }^{6}$ In this and the following plots for the $\mathrm{xSM}$, we use the same set of data points as used in Ref. [42].
}

lower (larger) than that in the GM model, which results in a more detectable GW signal. The triple scalar couplings has similar enhancement as in the GM model.

As a comparison, in Fig. 10, we show the two crucial parameters for the GW signals in both GM and xSM. The left panel indicates the scenario with extra EWSB contribution (GM). Since the extra EWSB contribution is subject to severe bounds from the LHC, the extra VEV is small which limits the vacuum structure that is important for the phase transition, and thus the first step of the phase transition is weakly first order and the GW signal being generated is negligible (with a small $\alpha$ and large $\beta / H_{n}$ ), the GW signals in this case mostly dominated by the secondstep SFOEWPT which characterize the dynamical EWSB. The right panel is for the case without extra EWSB (xSM); one may find that the $\alpha$ and $\beta / H_{n}$ of the first-step phase transition are mostly smaller than the second-step, which implies that a GW signal mostly comes from the first-step which does not characterize any symmetry breaking; see Ref. [42] for more details of the phase transition in xSM. 

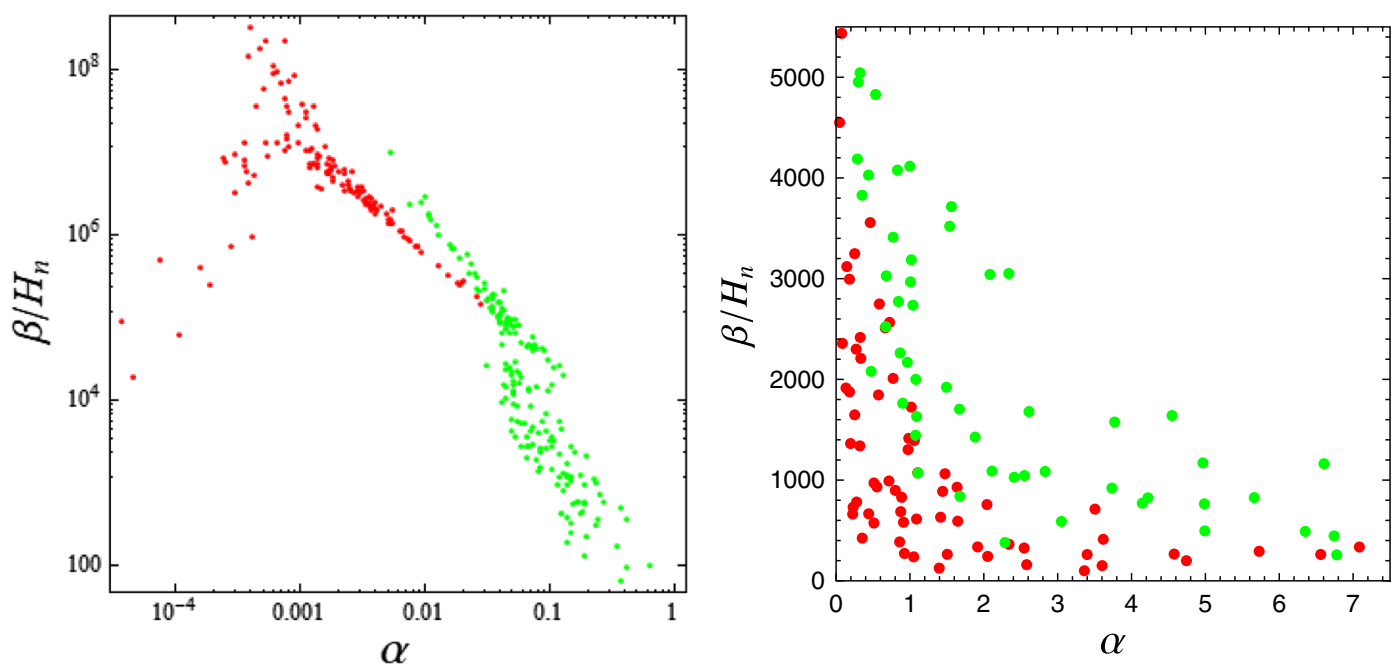

FIG. 10. The GW signal parameters for the case with extra EWSB (GM, left panel) and without extra EWSB (xSM, right panel). The red and green points correspond to the first and the second step of the two-step SFOEWPT.

There are mainly two sources for GW production during the EWPT: the sound waves in the plasma $[56,57]$ and the magnetohydrodynamic turbulence (MHD) [56,57], while the contribution from bubble wall collisions [58-63] is now generally believed to be negligible [64]. The energy density spectrum from the sound waves can be well expressed by [57]

$$
\begin{aligned}
\Omega_{\mathrm{sw}} h^{2}= & 2.65 \times 10^{-6}\left(\frac{H_{*}}{\beta}\right)\left(\frac{\kappa_{v} \alpha}{1+\alpha}\right)^{2}\left(\frac{100}{g_{*}}\right)^{1 / 3} \\
& \times v_{w}\left(\frac{f}{f_{\mathrm{sw}}}\right)^{3}\left(\frac{7}{4+3\left(f / f_{\mathrm{sw}}\right)^{2}}\right)^{7 / 2},
\end{aligned}
$$

where $H_{*}$ is the Hubble parameter at the temperature $T_{*}$, at the time when the EWPT finishes; $v_{w}$ is the bubble wall velocity; $\alpha$ is the energy released from the EWPT normalized by the total radiation energy density at $T_{*}$, as mentioned above; $g_{*}$ is the corresponding relativistic d.o.f. making up the radiation energy density; $\beta$ characterizes roughly the inverse time duration of the EWPT. Practically, $T_{*}$ is very close to $T_{n}$ and we use $T_{n}$ in the following calculations. Moreover $\kappa_{v}$ is the fraction of released energy going to the kinetic energy of the plasma, which can be calculated given $v_{w}$ and $\alpha$ [65]. Finally $f_{\text {sw }}$ is the peak frequency of the above energy density spectrum:

$$
f_{\mathrm{sw}}=1.9 \times 10^{-5} \frac{1}{v_{w}}\left(\frac{\beta}{H_{*}}\right)\left(\frac{T_{*}}{100 \mathrm{GeV}}\right)\left(\frac{g_{*}}{100}\right)^{1 / 6} \mathrm{~Hz} .
$$

A small fraction of the energy goes to the MHD, whose contribution to the energy density spectrum can be expressed as $[66,67]$

$$
\begin{aligned}
\Omega_{\mathrm{turb}} h^{2}= & 3.35 \times 10^{-4}\left(\frac{H_{*}}{\beta}\right)\left(\frac{\kappa_{\mathrm{turb}} \alpha}{1+\alpha}\right)^{3 / 2}\left(\frac{100}{g_{*}}\right)^{1 / 3} \\
& \times v_{w} \frac{\left(f / f_{\text {turb }}\right)^{3}}{\left[1+\left(f / f_{\text {turb }}\right)\right]^{11 / 3}\left(1+8 \pi f / h_{*}\right)},
\end{aligned}
$$

where the factor $\kappa_{\text {turb }}$ is the fraction of energy transferred to the MHD turbulence and is given roughly by $\kappa_{\text {turb }} \approx \epsilon \kappa_{v}$ with $\epsilon \approx 5-10 \%$ [57]. We take here $\epsilon \approx 0.1$. Similar to $f_{\mathrm{sw}}$, $f_{\text {turb }}$ is the peak frequency for the spectrum from the MHD:

$$
f_{\text {turb }}=2.7 \times 10^{-5} \frac{1}{v_{w}}\left(\frac{\beta}{H_{*}}\right)\left(\frac{T_{*}}{100 \mathrm{GeV}}\right)\left(\frac{g_{*}}{100}\right)^{1 / 6} \mathrm{~Hz} .
$$

In using above results for GW spectra calculations, care must be taken when choosing the value of $v_{w}$. While a larger and generally supersonic $v_{w}$ can lead to stronger GW signals, it is dangerous for baryon asymmetry generation in the framework of EWBG, which is assumed in this work to be the cosmological context within which EWPT happened. The EWBG favors a subsonic $v_{w}$, with a typical value of 0.05 (see e.g., [68-72]). This raises a serious problem for models of EWBG to be able to generate strong enough GW signals. To deal with this conundrum, we follow Refs. $[9,42,73,74]$ by taking the plasma hydrodynamics into account and distinguish $v_{w}$ from the velocity used in EWBG calculations. This is due to the existence of three modes of fluid velocity profiles around the bubble wall: deflagration, detonation, and supersonic deflagration (see [65] for a recent combined analysis). For the modes of deflagration and supersonic deflagration, the fluid outside the bubble wall has a nonzero velocity profile, and thus when boosted to the wall frame, the fluid would head towards the wall with a velocity that is different from and 

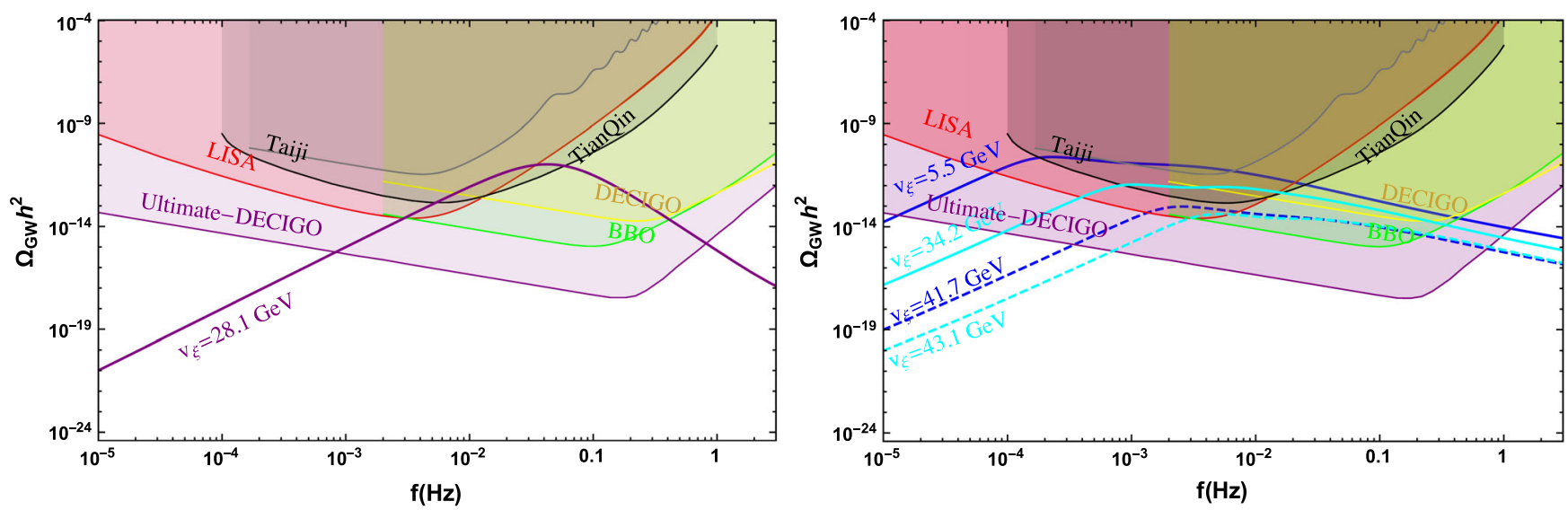

FIG. 11. The representative GW signal spectra for the cases of H5plane (left) and low mass benchmark (right). For the H5plane, only one-step SFOEWPT (purple) is available, while for the low mass benchmark, both one-step (cyan) and two-step (blue) transitions are available. The corresponding value of $v_{\xi}$ for each spectrum is also shown along each line.

smaller than the wall velocity. Therefore a supersonic $v_{w}$ can be realized while still maintaining a subsonic plasma velocity outside the bubble wall in the wall frame. While a joint analysis of the microscopic particle transport behavior within the EWBG and the macroscopic hydrodynamics is still needed to confirm the feasibility of the above mechanism, we assume tentatively that it is true here. With this in mind, we use $v_{w}=0.7$ for the Georgi-Machacek model. For the points we take from Ref. [42] for the xSM, a slightly different prescription was adopted there. There the value of $v_{w}$ was chosen such that a fixed value of 0.05 can be achieved for the velocity used in EWBG calculations. For those with SNR $>10$, the resulting $v_{w}$ all have relativistic values and are close to 0.7 , so the behavior of the following plots for the xSM remains largely unchanged should one stick to a fixed value of 0.7 .

In Fig. 11, we show the GW signal spectrum predicted in the H5plane (left) and the low mass benchmark (right) after considering the same-sign diboson bounds from CMS for several representative points taken from the tail of Fig. 7 and Fig. 8 (which are the most promising points for GW detection). The figure indicates that the magnitude of the GW spectra from the SFOEWPT of the H5plane and the low mass benchmark falls into the sensitivity curve of the LISA, and the peak frequency of the low mass benchmark is much lower than that of the H5plane.

With the GW spectrum obtained for each set of parameters input, the GW signals can be searched for using the cross correlation between the outputs of a pair of detectors. The detectability of the signals is quantified by the signalto-noise ratio (SNR) [51]:

$$
\mathrm{SNR}=\sqrt{\mathcal{T} \int d f\left[\frac{h^{2} \Omega_{\mathrm{GW}}(f)}{h^{2} \Omega_{\exp }(f)}\right]^{2}},
$$

where $\mathcal{T}$ is the mission duration in years and $\Omega_{\exp }$ is the power spectral density of a given detector.

\section{COLLIDER SEARCHES}

From previous studies (e.g., Fig. 9 of Ref. [21]), we find that one-step and two-step phase transition would happen in different parameter space regions. In the low mass benchmark, current constraints from same-sign diboson searches already separated the one-step and two-step phase transitions into two almost nonoverlapping parts with twostep points preferring lower mass while one-step preferred higher mass. Further, the phase transition strength has a correlation with the triple Higgs coupling in the one-step case, while it is not necessary in the two-step case to have larger triple Higgs coupling to trigger the SFOEWPT.

On the other hand, the collider searches of the Higgs potential also concentrate on the triple Higgs coupling searches: Higgs pairs search at hadron colliders (LHC, HLLHC, SppC, FCC-hh,etc.), and Zhh production at lepton colliders (ILC, CEPC, FCC-ee, etc.). Further, the quartic Higgs coupling also enters Higgs pair production through either two-loop [75] or one-loop [76] contributions for hadron and lepton colliders, respectively. Hence the Higgs pair production searches will be nice places to further search for these two cases at either the LHC or future lepton colliders.

The leading order contributions for Higgs pair production come from the one-loop diagrams shown in Fig. 12 with nonresonant and possible resonant productions. The nonresonant productions involve the box diagrams (right panel) and also triangle diagram (left panel) which depends on the triple scalar coupling $\lambda_{h h h}$. While the resonant production involves the production of extra scalar and subsequent decays into the Higgs pair (middle panel) which depends on the triple scalar coupling $\lambda_{H h h}$. The differential cross section for the Higgs pair production has been carried 


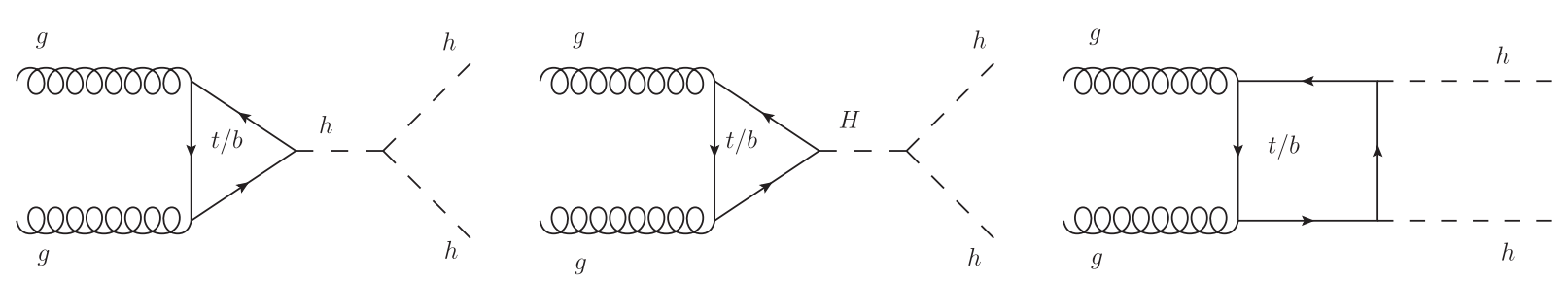

FIG. 12. The Feynman diagrams for the Higgs pair production with extra scalar at the LHC.

out previously $[77,78]$. The calculations have been implemented into MadGraph for several different cases [79]. For the GM model, we use the NLO UFO model files [80] implemented with FeynRules [81,82] using MadGraph [45] to directly calculate the cross section for relevant processes. The required parameter cards are generated using GMCalc 1.4.1 [32]. Relevant work can also be found in [83].

The Higgs pair production cross sections in the GM model are shown in the upper-left panels of the cases of Fig. 13 for H5plane and low mass benchmark. All points shown in these plots have passed the constraint from the CMS same-sign diboson searches $[21,27]$. For the case of
H5plane, only one-step phase transition exists (dark red points), while both one-step (red points) and two-step (blue points) phase transitions can happen for the case of low mass benchmark. For the two-step points (blue points) in low mass benchmark, we also separate them into two categories: "bubble one-step" and "bubble two-step", the same as those in Fig. 8. In either case, current Higgs pair searches do not have sufficient sensitivity to probe the phase transition viable parameter space. However, with accumulated data from HLLHC, it is possible to cover most points in H5plane, while points in low mass benchmark are still beyond the Higgs pair production measurements.
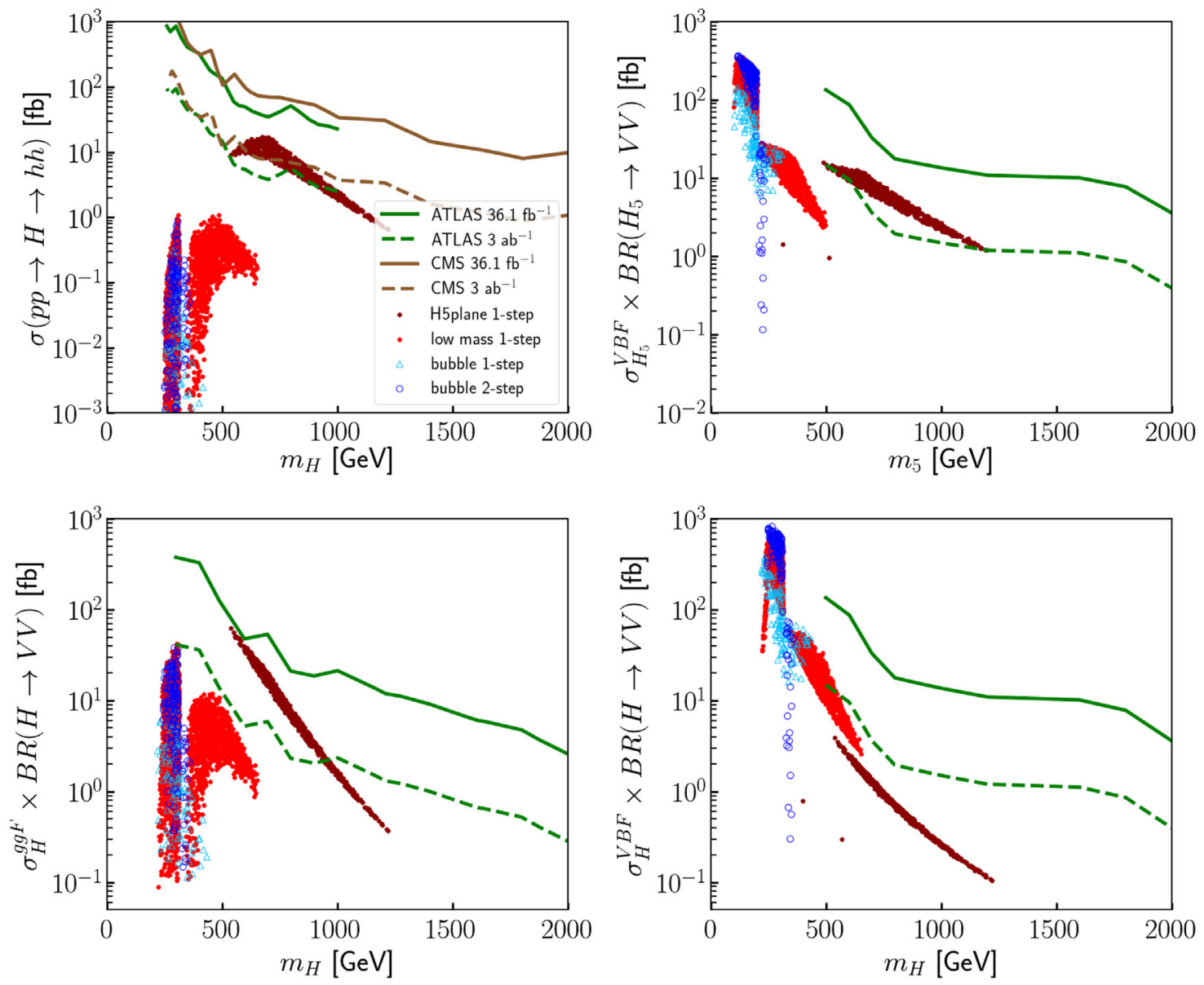

FIG. 13. The current and prospective di-Higgs and diboson searches at the LHC for GM model in the H5plane (dark red for one-step) and low mass benchmark (red and blue for one-step and two-step, respectively). The blue points are further separated into two categories: "bubble one-step" (light blue triangle) and "bubble two-step" (dark blue circle). 

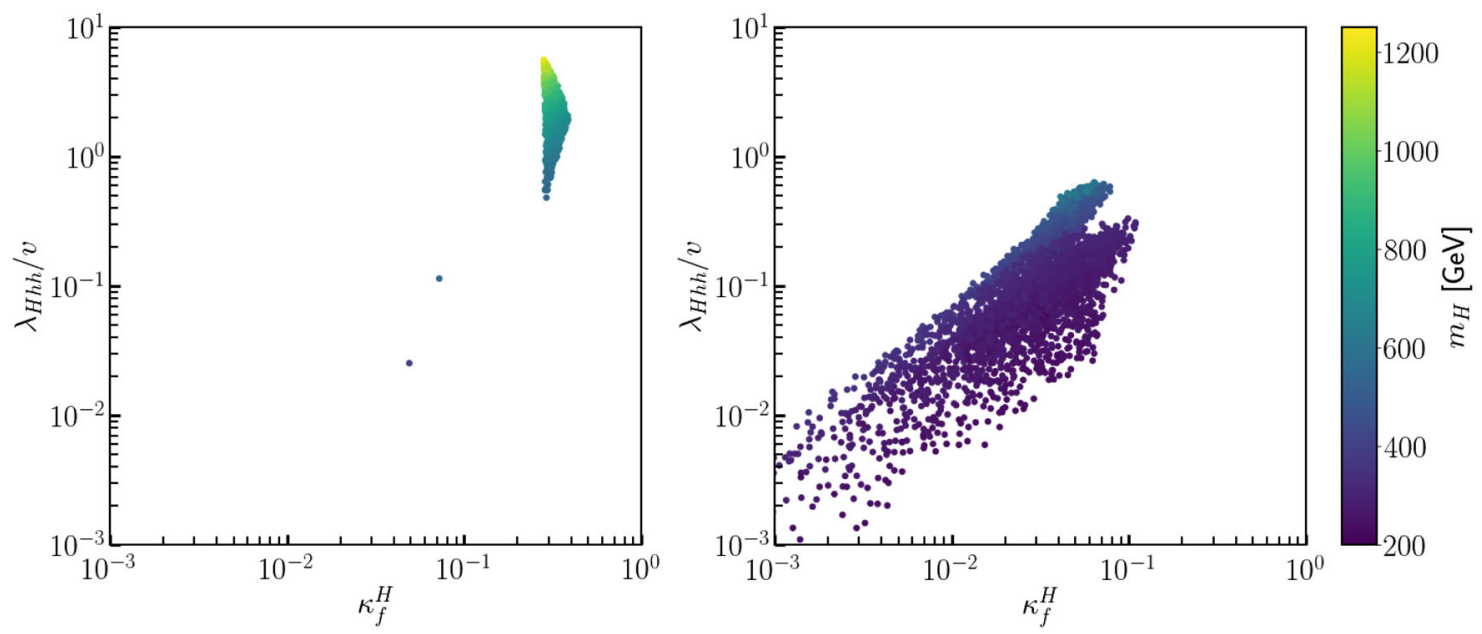

FIG. 14. SFOEWPT viable points in the $\kappa_{f}^{H}-\lambda_{H h h}$ plane for H5plane (left) and low mass benchmark (right). The colors of the points represent the mass of $H$.

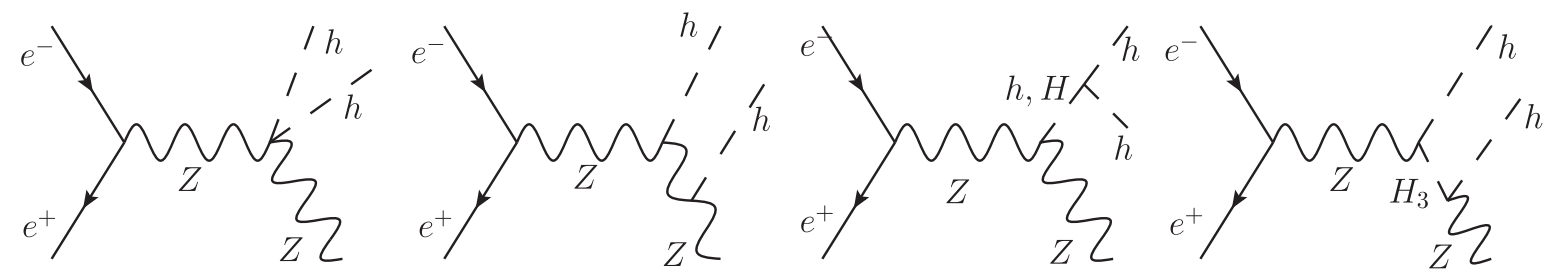

FIG. 15. The $h h Z$ production at the lepton collider in the GM model.

This can be understood from Fig. 14 in which we show the same points in $\lambda_{H h h}-\mathrm{BR}(H \rightarrow h h)$ plane for H5plane (left panel) and low mass benchmark (right panel). It is clear that in H5plane, which only has viable one-step points, the triple scalar coupling $\lambda_{H h h}$ is much larger than those in low mass benchmark. Hence, the branching of $H \rightarrow h h$ will be larger in H5plane. On the other hand, the $\kappa_{f}^{H} \equiv \frac{g_{H f f}^{\mathrm{GM}}}{g_{h f f}^{\mathrm{SM}}}=\frac{\sin \alpha_{H}}{\cos \theta_{H}}$, which contributes to the gluon-gluon fusion cross section, is also larger in H5plane than that in low mass benchmark. As a consequence, the cross section of the resonance Higgs pair production is larger in H5plane.

Aside from the Higgs pair measurements, the diboson resonance searches will also have the sensitivity to probe the phase transition viable parameter space. Hence, in both H5plane as well as low mass benchmark, we check the resonance diboson cross section through either $H$ (gluongluon Fusion and VBF) or $H_{5}(\mathrm{VBF})$ against the experimental limits. The results are shown in the other three panels in Fig. 13. From these plots, we find that, H5plane can be fully covered by the diboson searches from $\mathrm{H}_{5}$ (VBF) and $H$ gluon-gluon fusion $(\mathrm{ggF})$ resonance production, while low mass benchmark can still escape the searches. However, due to the large $\kappa_{V}^{H}$ compared with $\kappa_{f}^{H}$, the VBF production of $H$ is not highly suppressed. Thus extending the relevant searches into the lower mass region (below $500 \mathrm{GeV}$ ) will tremendously improve the sensitivity for this case.

There are also proposals focusing on the electron colliders aiming at the Higgs properties measurements. These lepton colliders also provide another opportunity to search for the Higgs pair production [84]. Hence, we also investigate the sensitivity of the Higgs pair production at the lepton collider associated with Z-boson for H5plane and low mass benchmark. The corresponding processes are shown in Fig. 15. The cross sections are calculated using MadGraph and the same model files as mentioned above. The SM cross section of such a process peaks at around $\sqrt{s}=500 \mathrm{GeV}$. To maximize the possible sensitivity, we thus focus on the $500 \mathrm{GeV}$ scenario of the lepton collider.

The unpolarized total cross sections are shown in Fig. 16 for H5plane (left panel) and low mass benchmark (right panel). The enhancement factor $\kappa=\frac{\sigma^{\mathrm{GM}}}{\sigma^{\mathrm{SM}}}$ is also indicated in the secondary y-axis on the right-hand side of each plot. We find that in H5plane, the cross section has a moderate enhancement with $\kappa \sim 2$ for all the viable points. However, in low mass benchmark, $\kappa$ spans a large range, and can even reach about 30 for a mass around $300 \mathrm{GeV}$. For H5plane, the moderate enhancement mainly comes from the large $\lambda_{h h h}$, as the collision energy is not enough for the resonance production through either $\mathrm{H}$ or $\mathrm{H}_{3}$. While, in the low mass benchmark, these masses are within the reach of the 

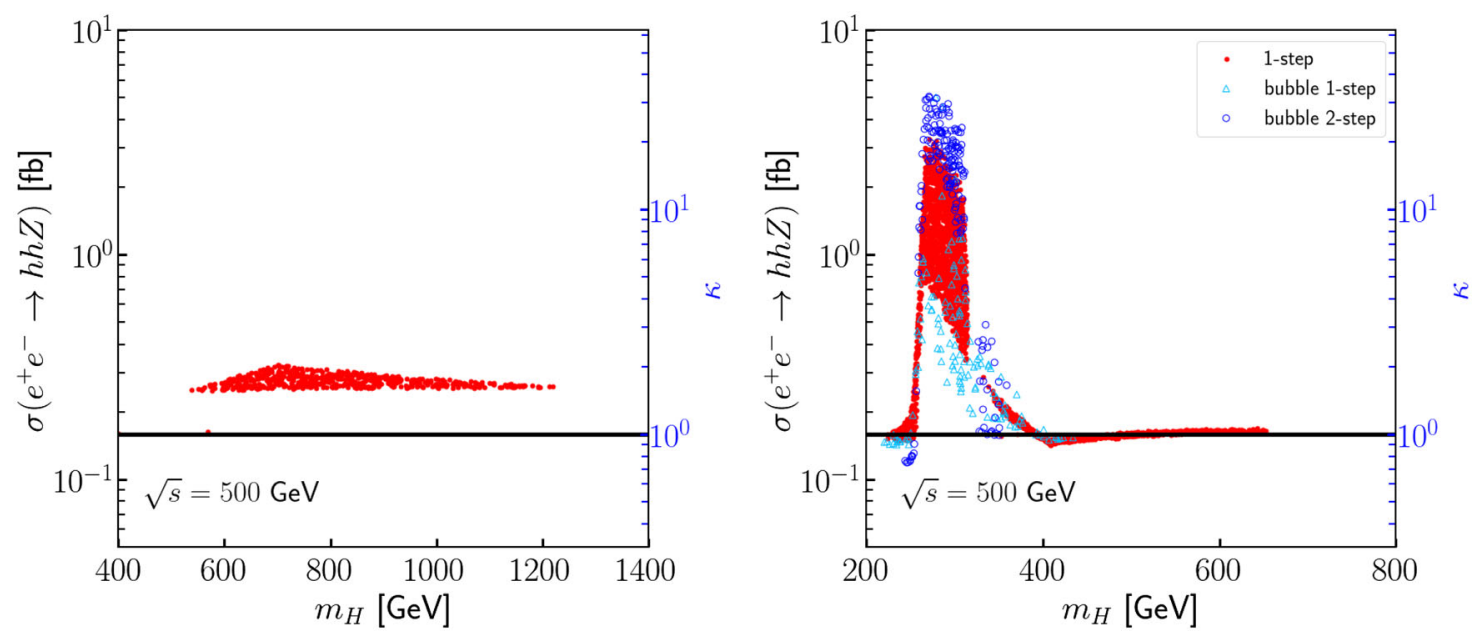

FIG. 16. The unpolarized cross section of $e^{+} e^{-} \rightarrow h h Z$ at $\sqrt{s}=500 \mathrm{GeV}$ for H5plane (left) and low mass benchmark (right) with one-step (red points) and two-step (blue points) EWPT. The horizontal line indicates the SM value. The secondary y-axis on the righthand side of each plot indicates $\kappa \equiv \frac{\sigma^{\mathrm{GM}}}{\sigma^{\mathrm{SM}}}$. The blue points are further separated into two categories: "bubble one-step" (light blue triangle) and "bubble two-step" (dark blue circle).
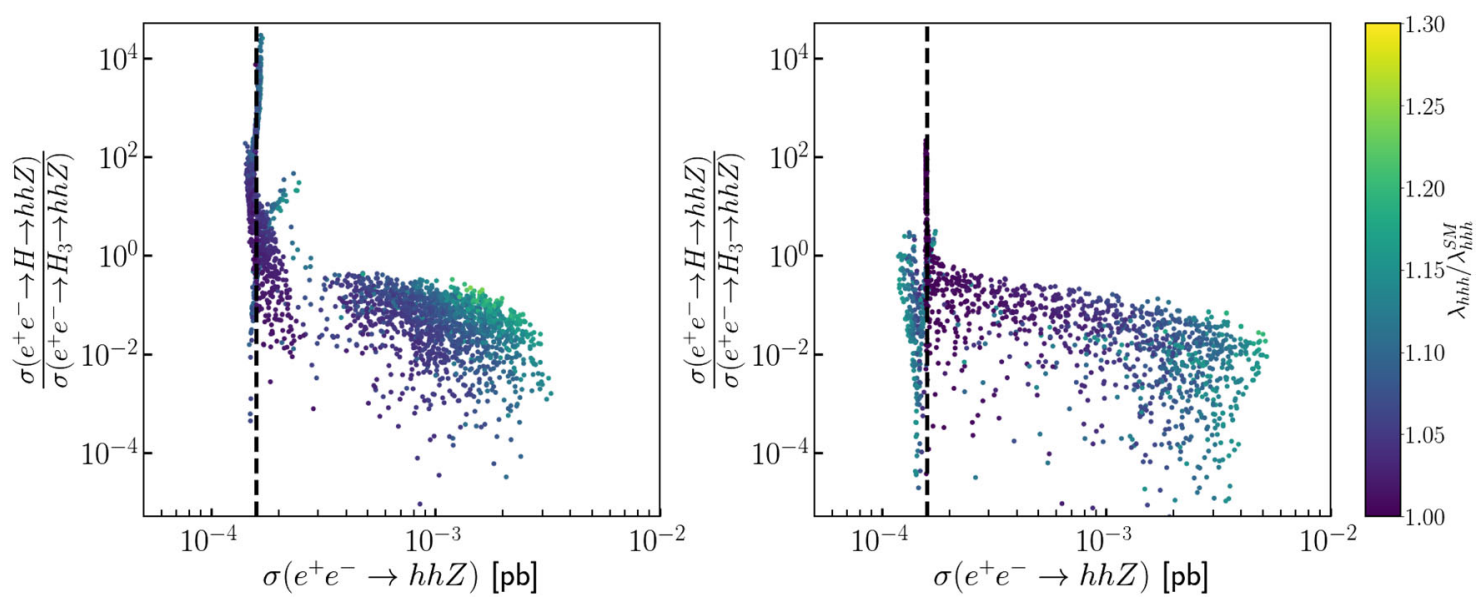

FIG. 17. The ratio between $H$ and $H_{3}$ induced the cross section with respect to the total cross section in low mass benchmark for onestep (left) and two-step (right) SFOEWPT.

collision energy, the resonance production of $\mathrm{H}$ and/or $\mathrm{H}_{3}$ induce the huge enhancement.

Figure 17 shows the ratio between $H$ and $H_{3}$ induced the cross section with respect to the total cross section in low mass benchmark for both one-step (left) and two-step (right) cases. It is clear that the enhancement in the total cross section mainly comes from $\mathrm{H}_{3}$ resonance in low mass benchmark. When the collision energy rises to even higher values, it is also possible, in H5plane, to enhance the Higgs pair production through $H_{3}$ and/or $H$ resonance. With the high precision that we can achieve for the cross section measurement at the ILC/CEPC, these machines will have sensitivity for these SFOEWPT viable points.

To give a more concrete sensitive study of such channels, the polarized cross sections are also calculated for $P\left(e^{-}, e^{+}\right)=(-80 \%, 30 \%)$ and $P\left(e^{-}, e^{+}\right)=(80 \%,-30 \%)$.
We combine the sensitivities from $b b b b$ and $b b W W$ channels of this process from $[85,86]$ to obtain the constraints in the $\sigma_{R L}-\sigma_{L R}$ plane [87] which is shown in Fig. 18. ${ }^{7}$ From this plot, we find that almost all the SFOEWPT viable points in H5plane can be excluded by this measurement. In the low mass benchmark, most points can also be excluded. However, we still have both one-step and two-step SFOEWPT viable points that are beyond the sensitivity in low mass benchmark.

Some other channels are also possible in complementing the GW signal studies, especially for the lower mass region. In [44], the authors studied the sensitivities from the

\footnotetext{
${ }^{7} \sigma_{L R}\left(\sigma_{R L}\right)$ denotes the cross section at beam polarization configurations of $\left(P_{e^{+}}, P_{e^{-}}\right)=(+1,-1)((-1,+1))$.
} 

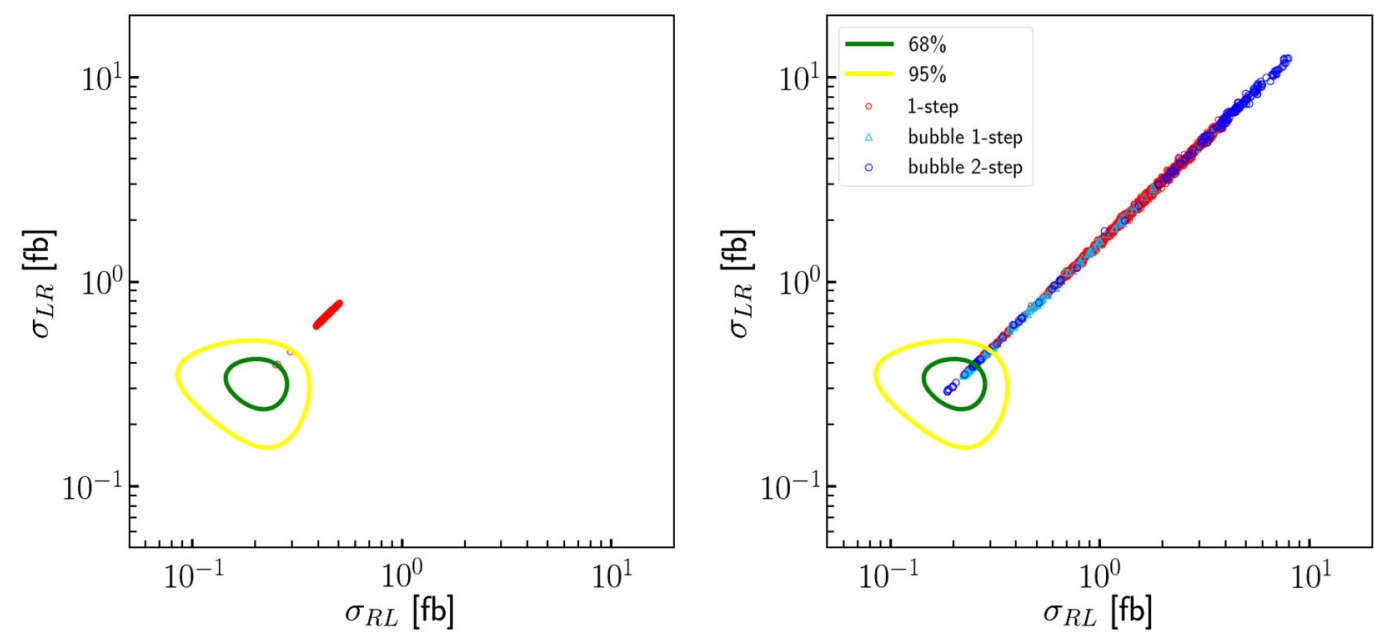

FIG. 18. The constraints of the polarized $h h Z$ production cross section measurement in the $\sigma_{R L}-\sigma_{L R}$ plane for $H 5 p l a n e$ (left) and low mass benchmark (right). The red and blue points are the one-step and two-step viable points in the GM parameter space, respectively. The green and yellow lines are the $68 \%$ and $95 \%$ C.L. contour, respectively. The blue points are further separated into two categories: "bubble one-step" (light blue triangle) and "bubble two-step" (dark blue circle).
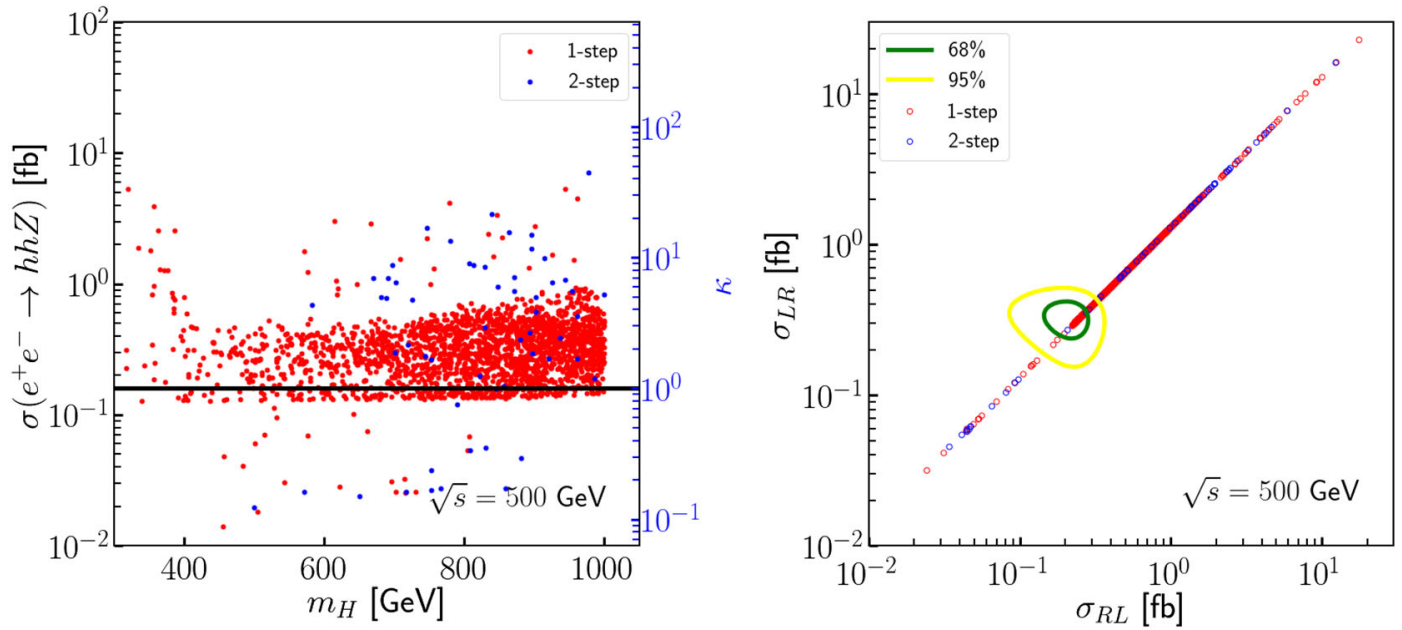

FIG. 19. The unpolarized cross section of $e^{+} e^{-} \rightarrow h h Z$ at $\sqrt{s}=500 \mathrm{GeV}$ (left) and the constraints of the polarized $h h Z$ production cross section measurement in the $\sigma_{R L}-\sigma_{L R}$ plane (right) for $\mathrm{xSM}$.

loop-induced channel $W \gamma$ from fermiophobic scalar which is specific for low mass benchmark. On the other hand, the diphoton searches [88,89] are also promising. However, after reinterpreting the bounds from [44], in our case, we found that they are not yet sensitive enough to reach the SFOEWPT viable points. Further improvements and detailed studies are needed for these searches.

In the $\mathrm{xSM}$, the high mass region of the extra Higgs is not going to be covered by Higgs pair production searches at the future HL-LHC, and a lot of parameter spaces there are not going to be probed by the diboson searches due to the small mixing angle of the SM Higgs and the heavy extra Higgs suppression effect. These regions can be complementarily searched for by the gravitational wave spacebased detectors [42]. At the ILC, the Higgs pair search results are shown in Fig. 19, where the cross sections are obtained using the same method as in the GM model. We find that in the xSM model, the cross sections (unpolarized or polarized) have moderate enhancement. From the prospects of the future cross section measurement, we could exclude most one-step as well as two-step points in the $\mathrm{xSM}$.

In Fig. 20, we show the sensitivities of future hadron and lepton colliders to the parameter spaces of one-step and two-step SFOEWPT for H5plane and low mass benchmark from the Higgs signal strength measurement, with the same conventions as those in Fig. 8. Here, all the curves are obtained by fitting the SM-like Higgs signal strength measurement from each experimental prospect (LHC [90-97], CEPC [98], ILC [99], and FCC-ee [100,101]). 

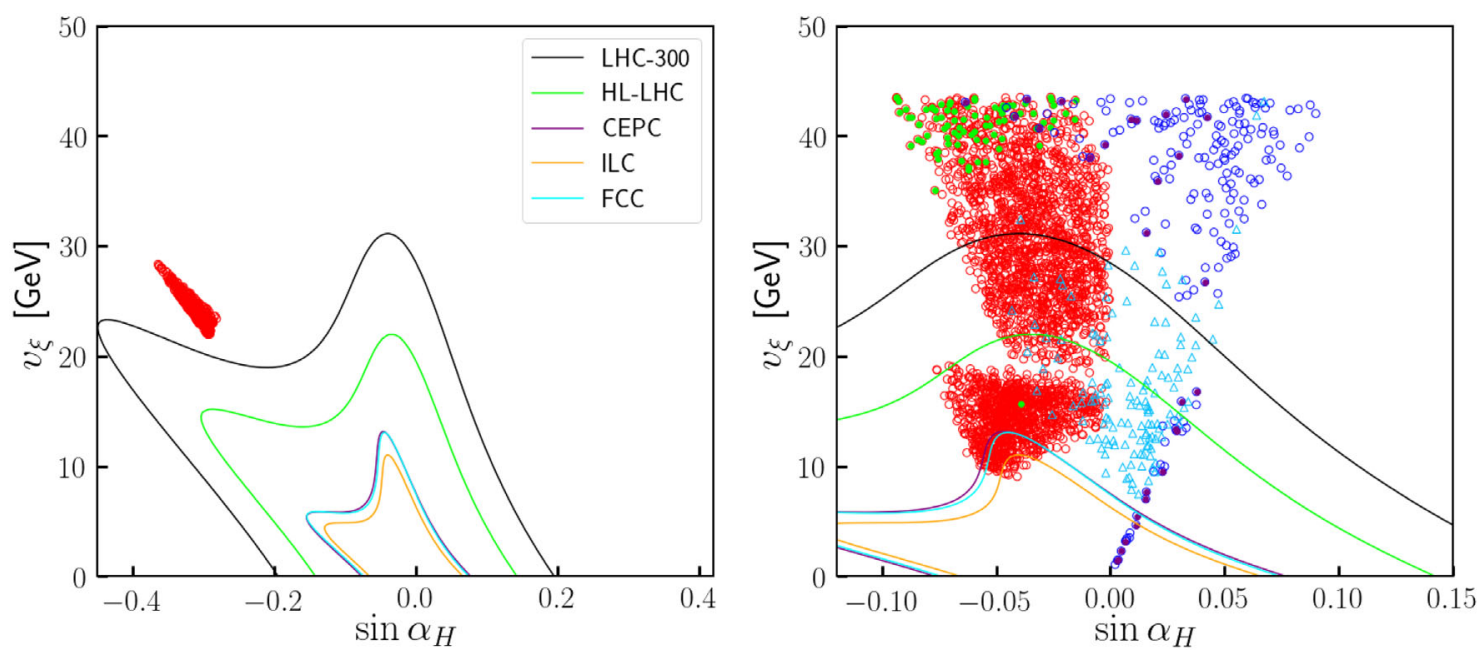

FIG. 20. The SFOEWPT viable points (bubble nucleation can occur and $v_{n} / T_{n}>1$ ) in the $\sin \alpha_{H}-v_{\xi}$ plane for one-step (red) and two-step (blue) phase transition for H5plane (left panel) and low mass benchmark (right panel) scenario. The contours with different colors represent the constraints from the Higgs precision measurement from different experiments as indicated in the legend. In low mass benchmark, we also indicate the points having SNR > 10 with solid markers for one-step (green) and two-step (purple) cases.

From Fig. 20, we find that most one-step points in both H5plane and low mass benchmark can be excluded by the Higgs signal strength measurements, though we still have a bunch of points for the two-step case located at around the alignment limit and escaping the constraint from the signal strength measurements. The stochastic GWs can be detected by finding the cross correlation of two independent interferometers (SNR) [51], we calculate this quantity for LISA in low mass benchmark. The SNR $>10$ points which are shown with filled points mostly concentrate in the region of a large $v_{\xi}$ for the one-step case. While for the two-step case, some points close to the alignment limit where the signal strength measurements have no sensitivity can have SNR $>10$. We note that the function of SNR (see Eq. (65) indicating lower sensitivity curves of detectors and a higher magnitude of the GW spectrum would generally lead to a larger value of SNR. We can expect that the Ultimate-DECIGO will generally have a larger SNR, while Taiji and TianQin will have a relatively lower SNR.

We finally present the sensitivities of future colliders in the SFOEWPT parameter space, with a conservative consideration where only the deviation of the SM couplings is included, with the $\delta \kappa_{3}$ and $\delta \kappa_{4}$ for the GM and xSM are given in Eq. (43) and Eq. (51). We first show in Fig. 21 the

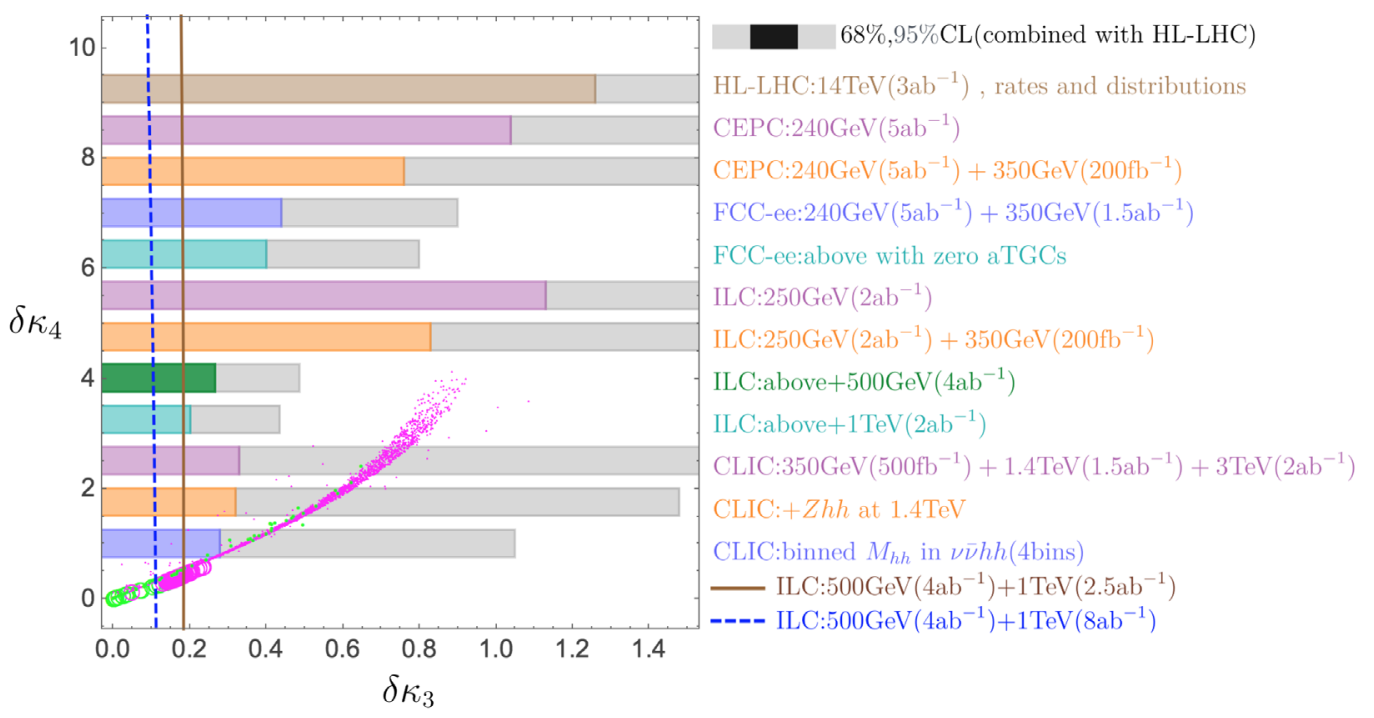

FIG. 21. Collider sensitivities of the triple Higgs coupling and quartic Higgs coupling with the SNR $>10$ points. The green and magenta points represent the two-step and one-step SFOEWPT cases. The circles and the dotted points represent the GM and xSM model scenarios. The bars and contours are the sensitivities taken from Refs. [76,102]. 
measurements of the two couplings $\left(\lambda_{h h h}\right.$ and $\left.\lambda_{h h h h}\right)$ at future $e^{+} e^{-}$colliders and the HL-LHC. Ref. [102] provides the $68 \% \mathrm{CL}$ and $95 \% \mathrm{CL}$ results (the inner and outer horizontal bar regions) of the future $e^{+} e^{-}$colliders and HLLHC measurements, though they only include the cubic coupling in their analysis based on an effective field theory approach. Another future ILC precision (brown and blue lines) is taken from Ref. [76], where the analysis is also based on an effective field theory approach and we expect the results for UV-complete models to have much better precision. For the situation with additional EWSB contributions, i.e., the GM model, we expect the future lepton hadron colliders to be more powerful, since the phase transition possibility would be highly restricted by collider searches, especially the diboson searches.

\section{CONCLUSIONS}

Utilizing the Georgi-Machacek (GM) model, we simultaneously study Higgs phenomenology and the electroweak phase transition for the scenario with the eEWSB contribution. Since the custodial symmetry allows cubic terms among Higgs doublets and isospin triplets, the eEWSB is found to be helpful to realize different vacuum structures from the SM as the Universe cools down, and therefore leads to different strongly first order electroweak phase transition patterns. We explored two benchmarks of the GM model to demonstrate this point: H5plane and low mass benchmark. We find that in H5plane, only one-step phase transition is allowed with relatively larger deviation of the triple Higgs coupling. Hence, the Higgs pair searches at the HL-LHC as well as the $h h Z$ production measurement at the lepton collider can cover most of the parameter space of H5plane, while in low mass benchmark, both one-step and two-step phase transitions are allowed. Compared with H5plane, the deviation of the triple Higgs coupling is much smaller. Thus, the Higgs pair searches at the LHC are not sensitive in this case, while the diboson searches are still possible when we extend the search range to the lower mass region. For $h h Z$ production at the lepton collider, instead of the enhancement from triple Higgs couplings, we have the resonance contributions from relatively light $\mathrm{H}_{3} / \mathrm{H}$ in low mass benchmark. Thus relevant measurements are also able to test most parameter space in low mass benchmark. For those points that can not be covered by collider searches, which are close to the alignment limit, the gravitational wave search provides a complementary way to probe the relevant parameter space.

For comparison, we also present the xSM model to show the situation without eEWSB. We found that: 1) in comparison with the GM, most of the SFOEWPT parameter space of the XSM is able to be covered by the future lepton colliders due to the relatively large mixing angle; 2) for the two-step scenario, the GWs come mostly from the secondstep SFOEWPT in the GM model and the first-step SFOEWPT in the XSM model; 3) in comparison with the $\mathrm{xSM}$ model, the GW signals from the SFOEWPT of the GM model require much higher precision of the colliders.

\section{ACKNOWLEDGMENT}

The work of L. G. B. is Supported by the National Natural Science Foundation of China (under Grants No. 11605016 and No. 11647307), the Fundamental Research Funds for the Central Universities of China (No. 2019CDXYWL0029). The work of Y. C. W. is partially supported by the Natural Sciences and Engineering Research Council of Canada. H. G. is supported in part by the U.S. Department of Energy Grant No. ds-sc0009956. We thank Yi Liao, Zhi-Long Han, and Bin Li for helpful discussions on the model. We also thank Tanmay Vachaspati, Alexander Vilenkin, Ken'ichi Saikawa for helpful communications and discussions on topological defects and related gravitational waves.

\section{APPENDIX A: EWPT IN THE SM + REAL SINGLET: XSM MODEL}

For the xSM model, the gauge invariant finite temperature effective potential is found to be $[38,42,103]$ :

$$
\begin{aligned}
V(h, s, T)=- & \frac{1}{2}\left[\mu^{2}-\Pi_{h}(T)\right] h^{2}-\frac{1}{2}\left[-b_{2}-\Pi_{s}(T)\right] s^{2} \\
& +\frac{1}{4} \lambda h^{4}+\frac{1}{4} a_{1} h^{2} s+\frac{1}{4} a_{2} h^{2} s^{2}+\frac{b_{3}}{3} s^{3}+\frac{b_{4}}{4} s^{4},
\end{aligned}
$$

with the thermal masses given by

TABLE I. XSM convention vs GM convention.

\begin{tabular}{lc}
\hline \hline $\mathrm{xSM}$ & $\mathrm{GM}$ \\
\hline$\frac{1}{24} T^{2}\left(a_{2}+3\left(3 g^{2}+g^{\prime 2}+2 Y_{t}^{2}+4 \lambda\right)\right)-\mu^{2}$ & $\frac{T^{2}}{8}\left(3 g^{2}+g^{2}+16 \lambda_{1}+12 \lambda_{4}+Y_{t}^{2} \sec \theta_{H}\right)+m_{1}^{2}$ \\
$b_{2}+\frac{1}{12}\left(2 a_{2}+3 b_{4}\right) T^{2}$ & $3 m_{2}^{2}+T^{2}\left(3 g^{2}+g^{\prime 2}+11 \lambda_{2}+7 \lambda_{3}+2 \lambda_{4}\right)$ \\
$\lambda$ & $4 \lambda_{1}$ \\
$b_{4}$ & $12\left(3 \lambda_{2}+\lambda_{3}\right)$ \\
$a_{2}$ & $6\left(2 \lambda_{4}+\lambda_{5}\right)$ \\
$a_{1}$ & $3 \mu_{1}$ \\
$b_{3}$ & $18 \mu_{2}$ \\
\hline \hline
\end{tabular}




$$
\begin{aligned}
\Pi_{h}(T) & =\left(\frac{2 m_{W}^{2}+m_{Z}^{2}+2 m_{t}^{2}}{4 v^{2}}+\frac{\lambda}{2}+\frac{a_{2}}{24}\right) T^{2}, \\
\Pi_{s}(T) & =\left(\frac{a_{2}}{6}+\frac{b_{4}}{4}\right) T^{2} .
\end{aligned}
$$

Identifying the coefficient for terms with the same power of different fields, we can get the correspondence between the parameters in the GM and $\mathrm{xSM}$, which is listed in Table I.

\section{APPENDIX B: THE $h^{6}$ OPERATOR FOR GM AND XSM MODELS}

The dimensional six operator $h^{6}$ can modify both cubic and quartic Higgs couplings, with the Higgs potential for the Higgs boson given by

$$
V_{\mathrm{SM}+h^{6}}^{h}=-\frac{\mu_{h}^{2}}{2} h^{2}+\frac{\lambda_{h}}{4} h^{4}+\frac{1}{8 \Lambda^{2}} h^{6} .
$$

Here, the $\Lambda$ indicates the scale where the heavy particles are integrated out. The minimization conditions lead to the following relations:

$$
\mu_{h}^{2}=\frac{m_{h}^{2}}{2}-\frac{3 v^{4}}{4 \Lambda^{2}}, \quad \lambda=\frac{m_{h}^{2}}{2 v^{2}}-\frac{3 v^{2}}{2 \Lambda^{2}} .
$$

In this scenario, the requirement of the EW minimum being the global minimum leads to

$$
\Lambda \geq v^{2} / m_{h}
$$
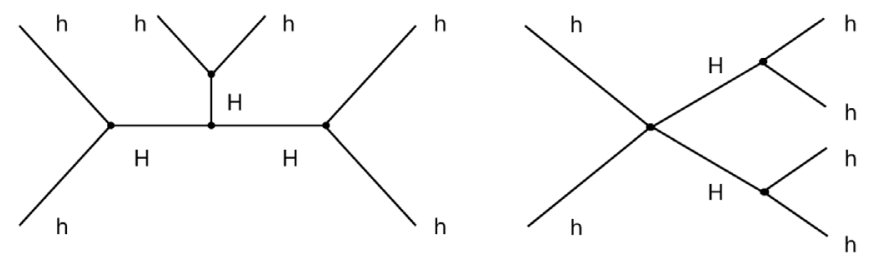

FIG. 22. The Feynman diagram for $\left(H^{\dagger} H\right)^{3}$ operator.

The cubic and quartic Higgs couplings are modified by the dimension- 6 operator as

$$
\lambda_{3 h}^{h^{6}}=\frac{3 m_{h}^{2}}{v}+\frac{6 v^{3}}{\Lambda^{2}}, \quad \lambda_{4 h}^{h^{6}}=\frac{3 m_{h}^{2}}{v^{2}}+\frac{36 v^{2}}{\Lambda^{2}} .
$$

The phase transition dynamics are estimated after taking into account the thermal correction of $c_{h}$, as in Ref. [47]. As studied in Refs. [46,47], to obtain a SFOEWPT with the $h^{6}$ operators, the additional contribution to the quartic Higgs coupling $\lambda$ should compensate its negative value and therefore ensure the possibility of the SFOEWPT. For related collider signals in synergy with gravitational waves study, we refer to Refs. [104-106]. See Refs. [107-109] for the GW and baryogenesis studies within the EFT framework with nonlinearly realized electroweak gauge symmetry, and Ref. [110] for a study of symmetry breaking patterns and gravitational waves in SMEFT with varying couplings.

Suppose the extra Higgs is much heavier than the SM Higgs; the $\left(H^{\dagger} H\right)^{3}$ operator can be obtained after integrating out the heavy Higgs in Fig. 22. For the GM model, the relevant interactions are given by

$$
\begin{aligned}
\lambda_{2 h H}^{\mathrm{GM}} & =\frac{\sqrt{3}}{2} \cos \alpha_{H}\left(\mu_{1} \cos \alpha_{H}^{2}-2 \sin \alpha_{H}^{2}\left(\mu_{1}-4 \mu_{2}\right)\right), \\
\lambda_{2 h 2 H}^{\mathrm{GM}} & =\cos 4 \alpha_{H}\left(-3 \lambda_{1}-3 \lambda_{2}-\lambda_{3}+3 \lambda_{4}+\frac{3 \lambda_{5}}{2}\right)+3 \lambda_{1}+3 \lambda_{2}+\lambda_{3}+\lambda_{4}+\frac{\lambda_{5}}{2}, \\
\lambda_{3 H}^{\mathrm{GM}} & =\frac{\sqrt{3}}{2} \cos \alpha_{H}\left(3 \mu_{1} \sin \alpha_{H}^{2}+8 \mu_{2} \cos \alpha_{H}^{2}\right) .
\end{aligned}
$$

Using the equation of motion on Eq. (40), the coefficients of $c_{4}, c_{6}, c_{8}$ for the GM are obtained as follows:

$$
\begin{aligned}
c_{4}^{\mathrm{GM}}= & \lambda_{1} \cos ^{4} \alpha_{H}+\sin ^{4} \alpha_{H}\left(\lambda_{2}+\frac{\lambda_{3}}{3}\right)+\lambda_{4} \sin ^{2} \alpha_{H} \cos ^{2} \alpha_{H}+\frac{1}{8} \lambda_{5} \sin ^{2} 2 \alpha_{H}-\frac{3 \cos ^{2} \alpha_{H}\left(\cos 2 \alpha_{H}\left(8 \mu_{2}-3 \mu_{1}\right)+\mu_{1}-8 \mu_{2}\right)^{2}}{128\left(m_{1}^{2} \sin ^{2} \alpha_{H}+m_{2}^{2} \cos ^{2} \alpha_{H}\right)}, \\
c_{6}^{\mathrm{GM}}= & \frac{1}{2048\left(m_{1}^{2} \sin ^{2} \alpha_{H}+m_{2}^{2} \cos ^{2} \alpha_{H}\right)^{3}} 3 \cos ^{2} \alpha_{H}\left(\cos 2 \alpha\left(8 \mu_{2}-3 \mu_{1}\right)+\mu_{1}-8 \mu_{2}\right)^{2} \\
& \times\left(4 ( m _ { 1 } ^ { 2 } \operatorname { s i n } ^ { 2 } \alpha _ { H } + m _ { 2 } ^ { 2 } \operatorname { c o s } ^ { 2 } \alpha _ { H } ) \left(\cos 4 \alpha_{H}\left(-6 \lambda_{1}-6 \lambda_{2}-2 \lambda_{3}+6 \lambda_{4}+3 \lambda_{5}\right)\right.\right. \\
& \left.\left.+6 \lambda_{1}+6 \lambda_{2}+2 \lambda_{3}+2 \lambda_{4}+\lambda_{5}\right)-\cos ^{2} \alpha_{H}\left(\cos 2 \alpha_{H}\left(3 \mu_{1}-8 \mu_{2}\right)-\mu_{1}+8 \mu_{2}\right)\left(3 \mu_{1} \sin ^{2} \alpha_{H}+8 \mu_{2} \cos ^{2} \alpha_{H}\right)\right), \\
c_{8}^{\mathrm{GM}}= & \frac{1}{32768\left(m_{1}^{2} \sin ^{2} \alpha_{H}+m_{2}^{2} \cos ^{2} \alpha_{H}\right)^{4}} 3 \cos ^{4} \alpha_{H}\left(\cos 2 \alpha_{H}\left(8 \mu_{2}-3 \mu_{1}\right)+\mu_{1}-8 \mu_{2}\right)^{4} \\
& \times\left(3\left(8 \lambda_{1} \sin ^{4} \alpha_{H}+\sin ^{2} 2 \alpha_{H}\left(2 \lambda_{4}+\lambda_{5}\right)\right)+8 \cos ^{4} \alpha_{H}\left(3 \lambda_{2}+\lambda_{3}\right)\right) .
\end{aligned}
$$

In the small mixing limit, the above relations reduce to 


$$
\begin{aligned}
c_{4}^{\mathrm{GM}}= & \lambda_{1}-\frac{3 \mu_{1}^{2}}{32 m_{2}^{2}}+\frac{\alpha_{H}^{2}\left(3 \mu_{1}^{2} m_{1}^{2}+16 m_{2}^{4}\left(-4 \lambda_{1}+2 \lambda_{4}+\lambda_{5}\right)+6 \mu_{1} m_{2}^{2}\left(3 \mu_{1}-8 \mu_{2}\right)\right)}{32 m_{2}^{4}}+O\left(\alpha_{H}^{3}\right), \\
c_{6}^{\mathrm{GM}}= & \frac{3 \mu_{1}^{2}\left(m_{2}^{2}\left(2 \lambda_{4}+\lambda_{5}\right)-\mu_{1} \mu_{2}\right)}{32 m_{2}^{6}}+\frac{3 \alpha_{H}^{2} \mu_{1}}{256 m_{2}^{8}}\left(24 \mu_{1}^{2} \mu_{2} m_{1}^{2}-\mu_{1} m_{2}^{2}\left(3\left(\mu_{1}^{2}-24 \mu_{1} \mu_{2}+64 \mu_{2}^{2}\right)\right.\right. \\
& \left.\left.+16 m_{1}^{2}\left(2 \lambda_{4}+\lambda_{5}\right)\right)+8 m_{2}^{4}\left(\mu_{1}\left(12 \lambda_{1}+12 \lambda_{2}+4 \lambda_{3}-11\left(2 \lambda_{4}+\lambda_{5}\right)\right)+16 \mu_{2}\left(2 \lambda_{4}+\lambda_{5}\right)\right)\right)+O\left(\alpha_{H}^{3}\right), \\
c_{8}^{\mathrm{GM}}= & \frac{3 \mu_{1}^{4}\left(3 \lambda_{2}+\lambda_{3}\right)}{256 m_{2}^{8}}+\frac{3 \alpha_{H}^{2} \mu_{1}^{3}}{512 \mathrm{~m} 2^{10}}\left(m_{2}^{2}\left(3 \mu_{1}\left(-24 \lambda_{2}-8 \lambda_{3}+2 \lambda_{4}+\lambda_{5}\right)+64 \mu_{2}\left(3 \lambda_{2}+\lambda_{3}\right)\right)\right. \\
& \left.-8 \mu_{1} m_{1}^{2}\left(3 \lambda_{2}+\lambda_{3}\right)\right)+O\left(\alpha_{H}^{3}\right) .
\end{aligned}
$$

The cubic and quartic couplings relevant for Fig. 22 in xSM are

$$
\begin{aligned}
\lambda_{2 h 2 H}^{\mathrm{XSM}} & =\frac{1}{4}\left(3\left(a_{2}-b_{4}-\lambda\right) \cos 4 \alpha_{H}+a_{2}+3\left(b_{4}+\lambda\right)\right), \\
\lambda_{3 H}^{\mathrm{XSM}} & =\frac{3}{2} a_{1} \sin \alpha_{H}^{2} \cos \alpha_{H}+2 b_{3} \cos \alpha_{H}^{3}, \\
\lambda_{2 h H}^{\mathrm{XSM}} & =\frac{1}{2} \cos \alpha_{H}\left(a_{1} \cos \alpha_{H}^{2}-2\left(a_{1}-2 b_{3}\right) \sin \alpha_{H}^{2}\right) .
\end{aligned}
$$

As in the GM model, one can use the equation of motion on Eq. (49) to obtain the coefficients of $c_{4}, c_{6}, c_{8}$ in xSM as follows:

$$
\begin{aligned}
c_{4}^{\mathrm{xSM}}= & \frac{1}{4}\left(a_{2} \sin ^{2} \alpha_{H} \cos ^{2} \alpha_{H}+b_{4} \sin ^{4} \alpha_{H}+\lambda \cos ^{4} \alpha_{H}\right)-\frac{\cos ^{2} \alpha_{H}\left(\left(4 b_{3}-3 a_{1}\right) \cos 2 \alpha_{H}+a_{1}-4 b_{3}\right)^{2}}{128\left(b_{2} \cos ^{2} \alpha_{H}-\mu^{2} \sin ^{2} \alpha_{H}\right)}, \\
c_{6}^{\mathrm{xSM}}= & \frac{1}{1024\left(b_{2} \cos ^{2} \alpha_{H}-\mu^{2} \sin ^{2} \alpha_{H}\right)^{3}} \cos ^{2} \alpha_{H}\left(\left(4 b_{3}-3 a_{1}\right) \cos 2 \alpha_{H}+a_{1}-4 b_{3}\right)^{2} \\
& \times\left(\left(3 \cos 4 \alpha_{H}\left(a_{2}-b_{4}-\lambda\right)+a_{2}+3\left(b_{4}+\lambda\right)\right)\left(b_{2} \cos ^{2} \alpha_{H}-\mu^{2} \sin ^{2} \alpha_{H}\right)\right. \\
& \left.-\frac{1}{6} \cos \alpha_{H}\left(\left(3 a_{1}-4 b_{3}\right) \cos 2 \alpha_{H}-a_{1}+4 b_{3}\right)\left(3 a_{1} \sin ^{2} \alpha_{H} \cos \alpha_{H}+4 b_{3} \cos ^{3} \alpha_{H}\right)\right), \\
c_{8}^{\mathrm{xSM}}= & \frac{\cos ^{4} \alpha_{H}\left(\left(4 b_{3}-3 a_{1}\right) \cos 2 \alpha_{H}+a_{1}-4 b_{3}\right)^{4}\left(a_{2} \sin ^{2} \alpha_{H} \cos ^{2} \alpha_{H}+b_{4} \cos ^{4} \alpha+\lambda \sin ^{4} \alpha_{H}\right)}{16384\left(b_{2} \cos ^{2} \alpha_{H}-\mu^{2} \sin ^{2} \alpha_{H}\right)^{4}} .
\end{aligned}
$$

In the small mixing limit, one has

$$
\begin{aligned}
c_{4}^{\mathrm{xSM}}= & -\frac{a_{1}^{2}-8 b_{2} \lambda}{32 b_{2}}+\frac{\alpha_{H}^{2}\left(a_{1}^{2}\left(6 b_{2}-\mu^{2}\right)-8 a_{1} b_{2} b_{3}+8 b_{2}^{2}\left(a_{2}-2 \lambda\right)\right)}{32 b_{2}^{2}}+O\left(\alpha_{H}^{3}\right), \\
c_{6}^{\mathrm{xSM}}= & -\frac{a_{1}^{2}\left(a_{1} b_{3}-3 a_{2} b_{2}\right)}{192 b_{2}^{3}}-\frac{\alpha_{H}^{2} a_{1}}{256 b_{2}^{4}}\left(a_{1}^{3} b_{2}+4 a_{1}^{2} b_{3}\left(\mu^{2}-3 b_{2}\right)\right. \\
& \left.+4 a_{1} b_{2}\left(a 2\left(11 b_{2}-2 \mu^{2}\right)-6 b_{2}(b 4+\lambda)+4 b_{3}^{2}\right)-32 a_{2} b_{2}^{2} b_{3}\right)+O\left(\alpha_{H}^{3}\right), \\
c_{8}^{\mathrm{XSM}}= & \frac{a_{1}^{4} b_{4}}{1024 b_{2}^{4}}+\frac{a_{1}^{3} \alpha_{H}^{2}}{1024 b_{2}^{5}}\left(a_{1}\left(a_{2} b_{2}+4 b_{4}\left(\mu^{2}-3 b_{2}\right)\right)+16 b_{2} b_{3} b_{4}\right)+O\left(\alpha_{H}^{3}\right) .
\end{aligned}
$$

As studied in Ref. [111], the additional contribution to the $h^{4}$ can be reached, which captures the residual effects of the high dimensional operators. 

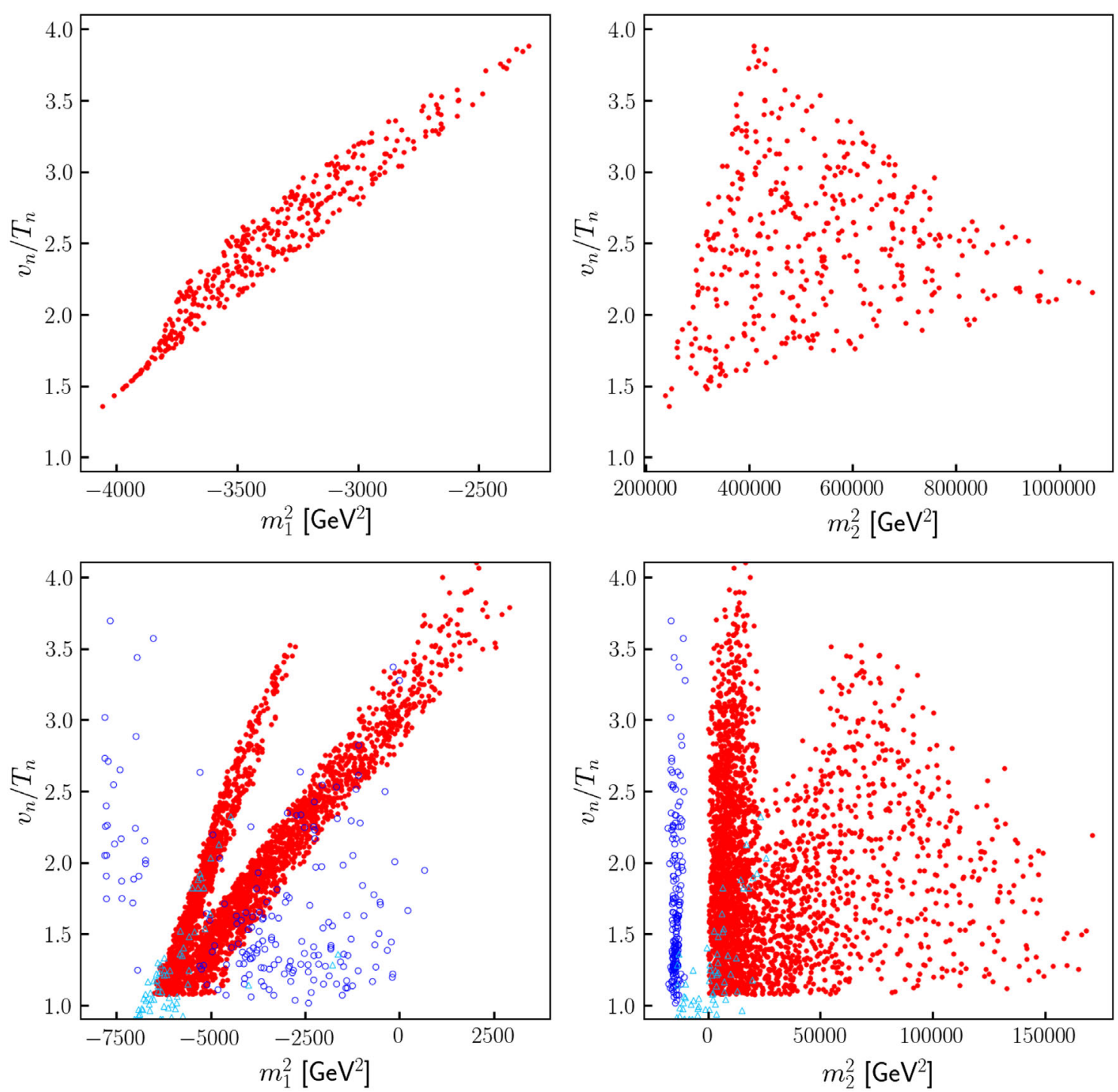

FIG. 23. The $m_{1}^{2}$ (left panels) and $m_{2}^{2}$ (right panels) distributions for H5plane (Top panels) and low mass benchmark (Bottom panels).
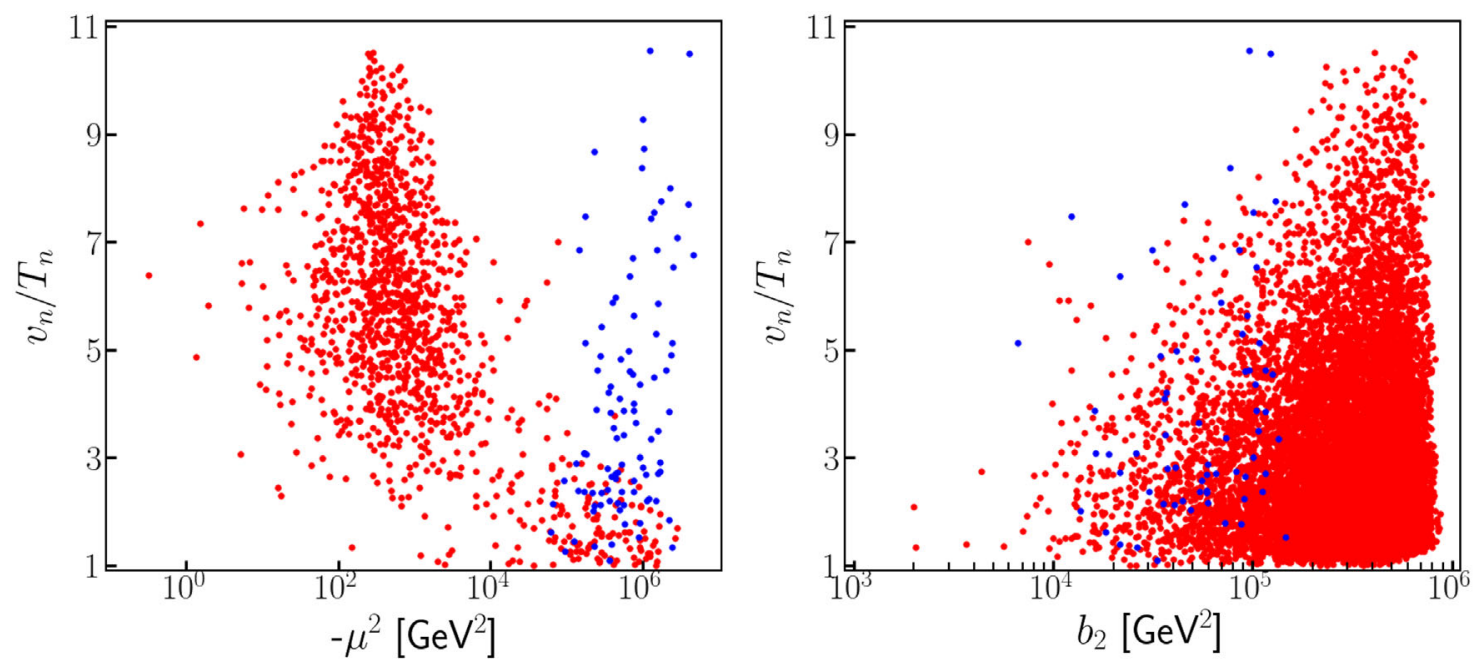

FIG. 24. The $\mu^{2}$ and $b_{2}$ distributions of the one-step and two-step SFOEWPT valid points for xSM. 
Up to now, we have calculated the high dimensional operators for the Higgs field with the method of CDE to match the UV models to the SMEFT [112]. These high dimensional operators, especially the $\left(H^{\dagger} H\right)^{3,4}$, are usually adopted to study phase transition in the literature. However, according to Fig. 23, which shows the distributions of $m_{1}^{2}$ and $m_{2}^{2}$ in both H5plane and low mass benchmark, the EFT approach does not apply for the GM model under study, especially the two-step case, as the basic assumption to integrate out the heavy d.o.f. is not fulfilled. The corresponding cases in XSM are also demonstrated in Fig. 24.

The fields of the light d.o.f. are usually necessary for the multistep phase transition; see Refs. $[9,12,16-18,20]$. In this situation, one could not employ the effective field theory (EFT) approach explored in the literature (e.g., $[46,47])$ to study the phase transition. Even for the one-step phase transition, there are also some mismatches between the EFT approach and the UV complete model studies, especially for the scenario where not so small mixing among the SM Higgs and extra Higgs is present (see the study of Ref. [113] for the "xSM" studies). We leave the detailed survey on the match for the phase transition dynamics between UV-complete models and the EFT approach to further studies. Indeed, the EFT approach for integrating out heavy degrees freedom is valid for the negligible mixing cases.

\section{APPENDIX C: SOME COUPLINGS IN GM MODEL}

$$
\begin{aligned}
& g_{H_{5}^{0} W^{-} W^{+}}=\frac{i e^{2} v \sin \left(\theta_{H}\right) \bar{g}^{\rho \sigma}}{2 \sqrt{3} s_{W}^{2}}, \quad g_{H_{5}^{0} Z Z}=-\frac{i e^{2} v \sin \left(\theta_{H}\right) \bar{g}^{\rho \sigma}}{\sqrt{3} c_{W}^{2} s_{W}^{2}}, \\
& g_{H_{5}^{\mp} W^{ \pm} Z}=-\frac{i e^{2} v \sin \left(\theta_{H}\right) \bar{g}^{\rho \sigma}}{2 c_{W} s_{W}^{2}}, \quad g_{H_{5}^{\mp \mp} W^{ \pm} W^{ \pm}}=\frac{i e^{2} v \sin \left(\theta_{H}\right) \bar{g}^{\rho \sigma}}{\sqrt{2} s_{W}^{2}}, \\
& g_{H W^{-} W^{+}}=\frac{i e^{2} v \sin \left(\alpha_{H}\right) \cos \left(\theta_{H}\right) \bar{g}^{\rho \sigma}}{2 s_{W}^{2}}+\frac{i \sqrt{\frac{2}{3}} e^{2} v \cos \left(\alpha_{H}\right) \sin \left(\theta_{H}\right) \bar{g}^{\rho \sigma}}{s_{W}^{2}}, \\
& g_{H Z Z}=\frac{i e^{2} v \sin \left(\alpha_{H}\right) \cos \left(\theta_{H}\right) \bar{g}^{\rho \sigma}}{2 c_{W}^{2} s_{W}^{2}}+\frac{i \sqrt{\frac{2}{3}} e^{2} v \cos \left(\alpha_{H}\right) \sin \left(\theta_{H}\right) \bar{g}^{\rho \sigma}}{c_{W}^{2} s_{W}^{2}}, \\
& g_{h W^{-} W^{+}}=\frac{i e^{2} v \cos \left(\alpha_{H}\right) \cos \left(\theta_{H}\right) \bar{g}^{\rho \sigma}}{2 s_{W}^{2}}-\frac{i \sqrt{\frac{2}{3}} e^{2} v \sin \left(\alpha_{H}\right) \sin \left(\theta_{H}\right) \bar{g}^{\rho \sigma}}{s_{W}^{2}}, \\
& g_{h Z Z}=\frac{i e^{2} v \cos \left(\alpha_{H}\right) \cos \left(\theta_{H}\right) \bar{g}^{\rho \sigma}}{2 c_{W}^{2} s_{W}^{2}}-\frac{i \sqrt{\frac{2}{3}} e^{2} v \sin \left(\alpha_{H}\right) \sin \left(\theta_{H}\right) \bar{g}^{\rho \sigma}}{c_{W}^{2} s_{W}^{2}}, \\
& g_{H_{3}^{0} h Z}=-\frac{\sqrt{\frac{2}{3}} e \sin \left(\alpha_{H}\right) \cos \left(\theta_{H}\right)}{c_{W} s_{W}}-\frac{e \cos \left(\alpha_{H}\right) \sin \left(\theta_{H}\right)}{2 c_{W} s_{W}}, \\
& \lambda_{H h h}=-\frac{2 i \sin \left(\alpha_{H}\right) \cos ^{2}\left(\alpha_{H}\right) m_{h}^{2} \sec \left(\theta_{H}\right)}{v}-\frac{2 i \sqrt{\frac{2}{3}} \sin \left(\alpha_{H}\right) \sin \left(2 \alpha_{H}\right) m_{h}^{2} \csc \left(\theta_{H}\right)}{v}-\frac{i \sin \left(\alpha_{H}\right) \cos ^{2}\left(\alpha_{H}\right) m_{H}^{2} \sec \left(\theta_{H}\right)}{v} \\
& -\frac{i \sqrt{\frac{2}{3}} \sin \left(\alpha_{H}\right) \sin \left(2 \alpha_{H}\right) m_{H}^{2} \csc \left(\theta_{H}\right)}{v}-2 i \sqrt{2} \mu_{1} \sin \left(\alpha_{H}\right) \cos ^{2}\left(\alpha_{H}\right) \cot \left(\theta_{H}\right)+i \sqrt{2} \mu_{1} \sin ^{3}\left(\alpha_{H}\right) \cot \left(\theta_{H}\right) \\
& -i \sqrt{3} \mu_{1} \sin \left(\alpha_{H}\right) \sin \left(2 \alpha_{H}\right) \cot ^{2}\left(\theta_{H}\right)+i \sqrt{3} \mu_{2} \sin \left(\alpha_{H}\right) \sin \left(2 \alpha_{H}\right), \\
& \lambda_{h h h}=-\frac{3 i \cos ^{3}\left(\alpha_{H}\right) m_{h}^{2} \sec \left(\theta_{H}\right)}{v}+\frac{2 i \sqrt{6} \sin ^{3}\left(\alpha_{H}\right) m_{h}^{2} \csc \left(\theta_{H}\right)}{v} \\
& +3 i \sqrt{2} \mu_{1} \sin ^{2}\left(\alpha_{H}\right) \cos \left(\alpha_{H}\right) \cot \left(\theta_{H}\right)+2 i \sqrt{3} \mu_{1} \sin ^{3}\left(\alpha_{H}\right) \cot ^{2}\left(\theta_{H}\right)-2 i \sqrt{3} \mu_{2} \sin ^{3}\left(\alpha_{H}\right) .
\end{aligned}
$$


[1] G. Aad et al. (ATLAS Collaboration), Observation of a new particle in the search for the Standard Model Higgs boson with the ATLAS detector at the LHC, Phys. Lett. B 716, 1 (2012).

[2] S. Chatrchyan et al. (CMS Collaboration), Observation of a new boson at a mass of $125 \mathrm{GeV}$ with the CMS experiment at the LHC, Phys. Lett. B 716, 30 (2012).

[3] B. P. Abbott et al. (LIGO Scientific and Virgo Collaborations), Observation of Gravitational Waves from a Binary Black Hole Merger, Phys. Rev. Lett. 116, 061102 (2016).

[4] B. P. Abbott et al. (LIGO Scientific and Virgo Collaborations), Upper Limits on the Stochastic Gravitational-Wave Background from Advanced LIGOs First Observing Run, Phys. Rev. Lett. 118, 121101 (2017); Erratum, Phys. Rev. Lett. 119, 029901 (2017).

[5] A. D. Sakharov, Violation of $C P$ invariance, C asymmetry, and baryon asymmetry of the universe, Pis'ma Zh. Eksp. Teor. Fiz. 5, 32 (1967); JETP Lett. 5, 24 (1967); Usp. Fiz. Nauk 161, 61 (1991); Sov. Phys. Usp. 34, 392 (1991).

[6] D. E. Morrissey and M. J. Ramsey-Musolf, Electroweak baryogenesis, New J. Phys. 14, 125003 (2012).

[7] A. Mazumdar and G. White, Cosmic phase transitions: Their applications and experimental signatures, Rep. Prog. Phys. 82, 076901 (2019).

[8] R. Zhou, L. Bian, and H.-K. Guo, Probing the electroweak sphaleron with gravitational waves, arXiv:1910.00234.

[9] A. Alves, T. Ghosh, H.-K. Guo, and K. Sinha, Resonant Di-Higgs production at gravitational wave benchmarks: A collider study using machine learning, J. High Energy Phys. 12 (2018) 070.

[10] G. C. Dorsch, S. J. Huber, K. Mimasu, and J. M. No, The Higgs vacuum uplifted: Revisiting the electroweak phase transition with a second Higgs doublet, J. High Energy Phys. 12 (2017) 086.

[11] C. P. D. Harman and S. J. Huber, Does zero temperature decide on the nature of the electroweak phase transition?, J. High Energy Phys. 06 (2016) 005.

[12] M. Jiang, L. Bian, W. Huang, and J. Shu, Impact of a complex singlet: Electroweak baryogenesis and dark matter, Phys. Rev. D 93, 065032 (2016).

[13] K. Ghorbani and P. H. Ghorbani, Strongly first-order phase transition in real singlet scalar dark matter model, J. Phys. G 47, 015201 (2020).

[14] L. Bian, H.-K. Guo, and J. Shu, Gravitational waves, baryon asymmetry of the universe and electric dipole moment in the $\mathrm{CP}$-violating NMSSM, Chin. Phys. C 42, 093106 (2018).

[15] J. Bernon, L. Bian, and Y. Jiang, A new insight into the phase transition in the early Universe with two Higgs doublets, J. High Energy Phys. 05 (2018) 151.

[16] W. Cheng and L. Bian, Higgs inflation and cosmological electroweak phase transition with $\mathrm{N}$ scalars in the postHiggs era, Phys. Rev. D 99, 035038 (2019).

[17] L. Bian and Y.-L. Tang, Thermally modified sterile neutrino portal dark matter and gravitational waves from phase transition: The Freeze-in case, J. High Energy Phys. 12 (2018) 006.

[18] L. Bian and X. Liu, Two-step strongly first-order electroweak phase transition modified FIMP dark matter, gravitational wave signals, and the neutrino mass, Phys. Rev. D 99, 055003 (2019).

[19] W. Chao, H.-K. Guo, and J. Shu, Gravitational wave signals of electroweak phase transition triggered by dark matter, J. Cosmol. Astropart. Phys. 09 (2017) 009.

[20] W. Cheng and L. Bian, From inflation to cosmological electroweak phase transition with a complex scalar singlet, Phys. Rev. D 98, 023524 (2018).

[21] R. Zhou, W. Cheng, X. Deng, L. Bian, and Y. Wu, Electroweak phase transition and Higgs phenomenology in the Georgi-Machacek model, J. High Energy Phys. 01 (2019) 216.

[22] A. Klein et al., Science with the space-based interferometer eLISA: Supermassive black hole binaries, Phys. Rev. D 93, 024003 (2016).

[23] H. Kudoh, A. Taruya, T. Hiramatsu, and Y. Himemoto, Detecting a gravitational-wave background with nextgeneration space interferometers, Phys. Rev. D 73, 064006 (2006).

[24] J. Luo et al. (TianQin Collaboration), TianQin: A spaceborne gravitational wave detector, Classical Quantum Gravity 33, 035010 (2016).

[25] X. Gong et al., Descope of the ALIA mission, J. Phys. Conf. Ser. 610, 012011 (2015).

[26] C.-W. Chiang and T. Yamada, Electroweak phase transition in Georgi? Machacek model, Phys. Lett. B 735, 295 (2014).

[27] A. M. Sirunyan et al. (CMS Collaboration), Observation of Electroweak Production of Same-Sign W Boson Pairs in the Two Jet and Two Same-Sign Lepton Final State in Proton-Proton Collisions at $\sqrt{s}=13 \mathrm{TeV}$, Phys. Rev. Lett. 120, 081801 (2018).

[28] A. Barroso, P. M. Ferreira, and R. Santos, Some remarks on tree-level vacuum stability in two Higgs doublet models, Afr. J. Math. Phys. 3, 103 (2006).

[29] A. Barroso, P. M. Ferreira, R. Santos, and J. P. Silva, Stability of the normal vacuum in multi-Higgs-doublet models, Phys. Rev. D 74, 085016 (2006).

[30] I. F. Ginzburg, K. A. Kanishev, M. Krawczyk, and D. Sokolowska, Evolution of Universe to the present inert phase, Phys. Rev. D 82, 123533 (2010).

[31] K. Hartling, K. Kumar, and H. E. Logan, The decoupling limit in the Georgi-Machacek model, Phys. Rev. D 90, 015007 (2014).

[32] K. Hartling, K. Kumar, and H. E. Logan, GMCALC: A calculator for the Georgi-Machacek model, arXiv: 1412.7387 .

[33] H. H. Patel and M. J. Ramsey-Musolf, Baryon washout, electroweak phase transition, and perturbation theory, J. High Energy Phys. 07 (2011) 029.

[34] M. Garny and T. Konstandin, On the gauge dependence of vacuum transitions at finite temperature, J. High Energy Phys. 07 (2012) 189.

[35] S. R. Coleman and E. J. Weinberg, Radiative corrections as the origin of spontaneous symmetry breaking, Phys. Rev. D 7, 1888 (1973).

[36] M. Quiros, Finite temperature field theory and phase transitions, in Proceedings, Summer School in Highenergy physics and cosmology: Trieste, Italy, 1998 (1999), pp. 187-259. 
[37] D. J. H. Chung, A. J. Long, and L.-T. Wang, $125 \mathrm{GeV}$ Higgs boson and electroweak phase transition model classes, Phys. Rev. D 87, 023509 (2013).

[38] S. Profumo, M. J. Ramsey-Musolf, C. L. Wainwright, and P. Winslow, Singlet-catalyzed electroweak phase transitions and precision Higgs boson studies, Phys. Rev. D 91, 035018 (2015).

[39] C. L. Wainwright, Cosmotransitions: Computing cosmological phase transition temperatures and bubble profiles with multiple fields, Comput. Phys. Commun. 183, 2006 (2012).

[40] C. Chatterjee, M. Kurachi, and M. Nitta, Topological defects in the Georgi-Machacek model, Phys. Rev. D 97, 115010 (2018).

[41] K. Saikawa, A review of gravitational waves from cosmic domain walls, Universe 3, 40 (2017).

[42] A. Alves, T. Ghosh, H.-K. Guo, K. Sinha, and D. Vagie, Collider and gravitational wave complementarity in exploring the singlet extension of the standard model, J. High Energy Phys. 04 (2019) 052.

[43] D. de Florian et al. (LHC Higgs Cross Section Working Group Collaboration), Handbook of LHC Higgs Cross Sections: 4. Deciphering the Nature of the Higgs Sector, http://dx.doi.org/10.23731/CYRM-2017-002.

[44] H. E. Logan and Y. Wu, Searching for the $W \gamma$ decay of a charged Higgs boson, J. High Energy Phys. 11 (2018) 121.

[45] J. Alwall, R. Frederix, S. Frixione, V. Hirschi, F. Maltoni, O. Mattelaer, H. S. Shao, T. Stelzer, P. Torrielli, and M. Zaro, The automated computation of tree-level and nextto-leading order differential cross sections, and their matching to parton shower simulations, J. High Energy Phys. 07 (2014) 079.

[46] C. Grojean, G. Servant, and J. D. Wells, First-order electroweak phase transition in the standard model with a low cutoff, Phys. Rev. D 71, 036001 (2005).

[47] P. Huang, A. Joglekar, B. Li, and C. E. M. Wagner, Probing the electroweak phase transition at the LHC, Phys. Rev. D 93, 055049 (2016).

[48] I. Affleck, Quantum Statistical Metastability, Phys. Rev. Lett. 46, 388 (1981).

[49] A. D. Linde, Decay of the false vacuum at finite temperature, Nucl. Phys. B216, 421 (1983); Erratum, Nucl. Phys. B223, 544 (1983).

[50] A. D. Linde, Fate of the false vacuum at finite temperature: Theory and applications, Phys. Lett. 100B, 37 (1981).

[51] C. Caprini et al., Science with the space-based interferometer eLISA. II: Gravitational waves from cosmological phase transitions, J. Cosmol. Astropart. Phys. 04 (2016) 001.

[52] M. Kamionkowski, A. Kosowsky, and M.S. Turner, Gravitational radiation from first order phase transitions, Phys. Rev. D 49, 2837 (1994).

[53] R. Apreda, M. Maggiore, A. Nicolis, and A. Riotto, Gravitational waves from electroweak phase transitions, Nucl. Phys. B631, 342 (2002).

[54] C. Grojean and G. Servant, Gravitational waves from phase transitions at the electroweak scale and beyond, Phys. Rev. D 75, 043507 (2007).

[55] S. J. Huber and T. Konstandin, Production of gravitational waves in the nMSSM, J. Cosmol. Astropart. Phys. 05 (2008) 017.
[56] M. Hindmarsh, S. J. Huber, K. Rummukainen, and D. J. Weir, Gravitational Waves from the Sound of a First Order Phase Transition, Phys. Rev. Lett. 112, 041301 (2014).

[57] M. Hindmarsh, S. J. Huber, K. Rummukainen, and D. J. Weir, Numerical simulations of acoustically generated gravitational waves at a first order phase transition, Phys. Rev. D 92, 123009 (2015).

[58] A. Kosowsky, M. S. Turner, and R. Watkins, Gravitational radiation from colliding vacuum bubbles, Phys. Rev. D 45, 4514 (1992).

[59] A. Kosowsky, M. S. Turner, and R. Watkins, Gravitational Waves from First Order Cosmological Phase Transitions, Phys. Rev. Lett. 69, 2026 (1992).

[60] A. Kosowsky and M.S. Turner, Gravitational radiation from colliding vacuum bubbles: Envelope approximation to many bubble collisions, Phys. Rev. D 47, 4372 (1993).

[61] S. J. Huber and T. Konstandin, Gravitational wave production by collisions: More bubbles, J. Cosmol. Astropart. Phys. 09 (2008) 022.

[62] R. Jinno and M. Takimoto, Gravitational waves from bubble collisions: An analytic derivation, Phys. Rev. D 95, 024009 (2017).

[63] R. Jinno and M. Takimoto, Gravitational waves from bubble dynamics: Beyond the envelope, J. Cosmol. Astropart. Phys. 01 (2019) 060.

[64] D. Bodeker and G. D. Moore, Can electroweak bubble walls run away?, J. Cosmol. Astropart. Phys. 05 (2009) 009.

[65] J. R. Espinosa, T. Konstandin, J. M. No, and G. Servant, Energy budget of cosmological first-order phase transitions, J. Cosmol. Astropart. Phys. 06 (2010) 028.

[66] C. Caprini, R. Durrer, and G. Servant, The stochastic gravitational wave background from turbulence and magnetic fields generated by a first-order phase transition, J. Cosmol. Astropart. Phys. 12 (2009) 024.

[67] P. Binetruy, A. Bohe, C. Caprini, and J.-F. Dufaux, Cosmological backgrounds of gravitational waves and eLISA/NGO: Phase transitions, cosmic strings and other sources, J. Cosmol. Astropart. Phys. 06 (2012) 027.

[68] P. John and M. G. Schmidt, Do stops slow down electroweak bubble walls?, Nucl. Phys. B598, 291 (2001); Erratum, Nucl. Phys. B648, 449 (2003).

[69] V. Cirigliano, S. Profumo, and M. J. Ramsey-Musolf, Baryogenesis, electric dipole moments and dark matter in the MSSM, J. High Energy Phys. 07 (2006) 002.

[70] D. J. H. Chung, B. Garbrecht, M. Ramsey-Musolf, and S. Tulin, Supergauge interactions and electroweak baryogenesis, J. High Energy Phys. 12 (2009) 067.

[71] W. Chao and M. J. Ramsey-Musolf, Electroweak baryogenesis, electric dipole moments, and Higgs diphoton decays, J. High Energy Phys. 10 (2014) 180.

[72] H.-K. Guo, Y.-Y. Li, T. Liu, M. Ramsey-Musolf, and J. Shu, Lepton-flavored electroweak baryogenesis, Phys. Rev. D 96, 115034 (2017).

[73] J. M. No, Large gravitational wave background signals in electroweak baryogenesis scenarios, Phys. Rev. D 84, 124025 (2011).

[74] A. Alves, D. Gonalves, T. Ghosh, H.-K. Guo, and K. Sinha, Di-Higgs production in the $4 b$ channel and gravitational wave complementarity, arXiv:1909.05268. 
[75] W. BizoA, U. Haisch, and L. Rottoli, Constraints on the quartic Higgs self-coupling from double-Higgs production at future hadron colliders, J. High Energy Phys. 10 (2019) 267.

[76] T. Liu, K.-F. Lyu, J. Ren, and H.X. Zhu, Probing the quartic Higgs boson self-interaction, Phys. Rev. D 98, 093004 (2018).

[77] O. J. P. Eboli, G. C. Marques, S. F. Novaes, and A. A. Natale, Twin Higgs boson production, Phys. Lett. B 197, 269 (1987).

[78] T. Plehn, M. Spira, and P. M. Zerwas, Pair production of neutral Higgs particles in gluon-gluon collisions, Nucl. Phys. B479, 46 (1996); Erratum, Nucl. Phys. B531, 655 (1998).

[79] B. Hespel and E. Vryonidou, https://cp3.irmp.ucl.ac.be/ projects/madgraph/wiki/HiggsPairProduction.

[80] A. Peterson, H. E. Logan, K. Hartling, K. Kumar, and Y. Wu, http://feynrules.irmp.ucl.ac.be/wiki/GeorgiMachacekModel.

[81] C. Degrande, Automatic evaluation of UV and R2 terms for beyond the Standard Model Lagrangians: A proof-ofprinciple, Comput. Phys. Commun. 197, 239 (2015).

[82] A. Alloul, N. D. Christensen, C. Degrande, C. Duhr, and B. Fuks, FeynRules 2.0-A complete toolbox for treelevel phenomenology, Comput. Phys. Commun. 185, 2250 (2014).

[83] J. Chang, C.-R. Chen, and C.-W. Chiang, Higgs boson pair productions in the Georgi-Machacek model at the LHC, J. High Energy Phys. 03 (2017) 137.

[84] B. Li, Z.-L. Han, and Y. Liao, Higgs production at future $e^{+} e^{-}$colliders in the Georgi-Machacek model, J. High Energy Phys. 02 (2018) 007.

[85] C.F. Durig, Measuring the Higgs self-coupling at the International Linear Collider, Ph.D. thesis, Hamburg University, Hamburg, 2016.

[86] M. Kurata and T. Tanabe, The Higgs self coupling analysis using the events containing $H \rightarrow W W^{*}$ Decay, Technical Report LC-REP-2013-025, 2013.

[87] L. Bian, N. Chen, W. Su, Y. Wu, and Y. Zhang, Future prospects of mass-degenerate Higgs bosons in the $\mathrm{CP}$ conserving two-Higgs-doublet model, Phys. Rev. D 97, 115007 (2018).

[88] G. Aad et al. (ATLAS Collaboration), Search for Scalar Diphoton Resonances in the Mass Range 65-600 GeV with the ATLAS Detector in $p p$ Collision Data at $\sqrt{s}=8$ TeV, Phys. Rev. Lett. 113, 171801 (2014).

[89] M. Aaboud et al. (ATLAS Collaboration), Search for new phenomena in high-mass diphoton final states using $37 \mathrm{fb}^{-1}$ of proton-proton collisions collected at $\sqrt{\mathrm{s}}=$ $13 \mathrm{TeV}$ with the ATLAS detector, Phys. Lett. B 775, 105 (2017).

[90] Projections for measurements of Higgs boson signal strengths and coupling parameters with the ATLAS detector at a HL-LHC, Technical Report ATL-PHYS-PUB2014-016, CERN, Geneva, 2014, http://cds.cern.ch/record/ 1956710.

[91] Projections for measurements of Higgs boson cross sections, branching ratios and coupling parameters with the ATLAS detector at a HL-LHC, Technical Report ATLPHYS-PUB-2013-014, CERN, Geneva, 2013, http://cds .cern.ch/record/1611186.
[92] HL-LHC projections for signal and background yield measurements of the $H \rightarrow \gamma \gamma$ when the Higgs boson is produced in association with $t$ quarks, $W$ or $Z$ bosons, Technical Report ATL-PHYS-PUB-2014-012, CERN, Geneva, 2014, http://cds.cern.ch/record/1741011.

[93] Update of the prospects for the $H \rightarrow Z \gamma$ search at the highluminosity LHC, Technical Report ATL-PHYS-PUB2014-006, CERN, Geneva, 2014, http://cds.cern.ch/ record/1703276.

[94] Prospects for the study of the Higgs boson in the VH(bb) channel at HL-LHC, Technical Report ATL-PHYS-PUB2014-011, CERN, Geneva, 2014, http://cds.cern.ch/record/ 1740962.

[95] Studies of the VBF $H \rightarrow \tau_{l} \tau_{\text {had }}$ analysis at high luminosity LHC conditions, Technical Report ATL-PHYS-PUB2014-018, CERN, Geneva, 2014, http://cds.cern.ch/ record/1956732.

[96] C. Hartmann and M. Trott, Higgs Decay to Two Photons at One Loop in the Standard Model Effective Field Theory, Phys. Rev. Lett. 115, 191801 (2015).

[97] ATLAS-Collaboration, Physics at a high-luminosity LHC with ATLAS (Update), Technical Report ATL-PHYSPUB-2012-004, CERN, Geneva, 2012, http://cds.cern .ch/record/1484890.

[98] M. Dong et al. (CEPC Study Group Collaboration), CEPC Conceptual Design Report: Volume 2-Physics \& Detector, arXiv:1811.10545.

[99] P. Bambade et al., The International Linear Collider: A Global Project, arXiv:1903.01629.

[100] A. Abada et al. (FCC Collaboration), FCC physics opportunities : Future circular collider conceptual design report Volume 1, Eur. Phys. J. C79, 474 (2019).

[101] A. Abada et al. (FCC Collaboration), FCC-ee: The lepton collider : Future circular collider conceptual design report Volume 2, Eur. Phys. J. 228, 261 (2019)

[102] S. Di Vita, G. Durieux, C. Grojean, J. Gu, Z. Liu, G. Panico, M. Riembau, and T. Vantalon, A global view on the Higgs self-coupling at lepton colliders, J. High Energy Phys. 02 (2018) 178.

[103] J. Kozaczuk, Bubble expansion and the viability of singletdriven electroweak baryogenesis, J. High Energy Phys. 10 (2015) 135.

[104] F. P. Huang, P.-H. Gu, P.-F. Yin, Z.-H. Yu, and X. Zhang, Testing the electroweak phase transition and electroweak baryogenesis at the LHC and a circular electron-positron collider, Phys. Rev. D 93, 103515 (2016).

[105] F. P. Huang, Y. Wan, D.-G. Wang, Y.-F. Cai, and X. Zhang, Hearing the echoes of electroweak baryogenesis with gravitational wave detectors, Phys. Rev. D 94, 041702 (2016).

[106] Q.-H. Cao, F. P. Huang, K.-P. Xie, and X. Zhang, Testing the electroweak phase transition in scalar extension models at lepton colliders, Chin. Phys. C 42, 023103 (2018).

[107] A. Kobakhidze, A. Manning, and J. Yue, Gravitational waves from the phase transition of a nonlinearly realized electroweak gauge symmetry, Int. J. Mod. Phys. D 26, 1750114 (2017).

[108] A. Kobakhidze, C. Lagger, A. Manning, and J. Yue, Gravitational waves from a supercooled electroweak phase 
transition and their detection with pulsar timing arrays, Eur. Phys. J. C 77, 570 (2017).

[109] A. Kobakhidze, L. Wu, and J. Yue, Electroweak baryogenesis with anomalous Higgs couplings, J. High Energy Phys. 04 (2016) 011.

[110] S. A. R. Ellis, S. Ipek, and G. White, Electroweak Baryogenesis from temperature-varying couplings, J. High Energy Phys. 08 (2019) 002.

[111] M. Jiang, N. Craig, Y.-Y. Li, and D. Sutherland, Complete one-loop matching for a singlet scalar in the
Standard Model EFT, J. High Energy Phys. 02 (2019) 031.

[112] B. Henning, X. Lu, and H. Murayama, How to use the Standard Model effective field theory, J. High Energy Phys. 01 (2016) 023.

[113] P. H. Damgaard, A. Haarr, D. O'Connell, and A. Tranberg, Effective field theory and electroweak baryogenesis in the singlet-extended standard model, J. High Energy Phys. 02 (2016) 107. 